\title{
Association of impaired neuronal migration with cognitive deficits in extremely preterm infants
}

Ken-ichiro Kubo, ${ }^{1}$ Kimiko Deguchi, ${ }^{1,2,3}$ Taku Nagai, ${ }^{4}$ Yukiko Ito, ${ }^{5}$ Keitaro Yoshida, ${ }^{6}$ Toshihiro Endo, ${ }^{7}$ Seico Benner, ${ }^{7}$ Wei Shan, ${ }^{4}$ Ayako Kitazawa, ${ }^{1,2}$ Michihiko Aramaki, ${ }^{1}$ Kazuhiro Ishii, ${ }^{1}$ Minkyung Shin, ${ }^{1}$ Yuki Matsunaga, ${ }^{1}$ Kanehiro Hayashi, ${ }^{1}$ Masaki Kakeyama, ${ }^{7,8}$ Chiharu Tohyama, ${ }^{7,}$ Kenji F. Tanaka, ${ }^{6}$ Kohichi Tanaka, ${ }^{5}$ Sachio Takashima, ${ }^{10}$ Masahiro Nakayama, ${ }^{11}$ Masayuki Itoh, ${ }^{2}$ Yukio Hirata, ${ }^{1}$ Barbara Antalffy, ${ }^{3}$ Dawna D. Armstrong, ${ }^{3}$ Kiyofumi Yamada, ${ }^{4}$ Ken Inoue, ${ }^{2}$ and Kazunori Nakajima

'Department of Anatomy, Keio University School of Medicine, Tokyo, Japan. ${ }^{2}$ Department of Mental Retardation and Birth Defect Research, National Institute of Neuroscience, National Center of Neurology and Psychiatry, Kodaira, Japan. ${ }^{3}$ Department of Pathology, Baylor College of Medicine, Houston, Texas, USA. ${ }^{4}$ Department of Neuropsychopharmacology and Hospital Pharmacy, Nagoya University Graduate School of Medicine, Nagoya, Japan. ${ }^{5}$ Department of Molecular Neuroscience, Medical Research Institute/School of Biomedical Science, Tokyo Medical and Dental University, Tokyo, Japan. ${ }^{6}$ Department of Neuropsychiatry, Keio University School of Medicine, Tokyo, Japan. 'Laboratory of Environmental Health Sciences, Center for Disease Biology and Integrative Medicine, Graduate School of Medicine, University of Tokyo, Tokyo, Japan. ${ }^{8}$ Laboratory for Systems Neuroscience \& Preventive Medicine, Waseda University Faculty of Human Sciences, Tokorozawa, Japan. ' $E$ Environmental Biology Laboratory, Faculty of Medicine, University of Tsukuba, Tsukuba, Japan. ${ }^{10}$ Division of Child Neurology, Yanagawa Institute of Developmental Disabilities, Yanagawa, Japan. "Department of Pathology, Osaka Medical Center and Research Institute for Maternal and Child Health, Izumi, Japan.

Many extremely preterm infants (born before 28 gestational weeks [CWs]) develop cognitive impairment in later life, although the underlying pathogenesis is not yet completely understood. Our examinations of the developing human neocortex confirmed that neuronal migration continues beyond $23 \mathrm{CWs}$, the gestational week at which extremely preterm infants have live births. We observed larger numbers of ectopic neurons in the white matter of the neocortex in human extremely preterm infants with brain injury and hypothesized that altered neuronal migration may be associated with cognitive impairment in later life. To confirm whether preterm brain injury affects neuronal migration, we produced brain damage in mouse embryos by occluding the maternal uterine arteries. The mice showed delayed neuronal migration, ectopic neurons in the white matter, altered neuronal alignment, and abnormal corticocortical axonal wiring. Similar to human extremely preterm infants with brain injury, the surviving mice exhibited cognitive deficits. Activation of the affected medial prefrontal cortices of the surviving mice improved working memory deficits, indicating that decreased neuronal activity caused the cognitive deficits. These findings suggest that altered neuronal migration altered by brain injury might contribute to the subsequent development of cognitive impairment in extremely preterm infants.

Authorship note: K. Kubo and K. Deguchi contributed equally to this work.

Conflict of interest: The authors have declared that no conflict of interest exists.

Submitted: May 16, 2016

Accepted: April 18, 2017

Published: May 18, 2017

Reference information: JCI Insight. 2017;2(10):e88609. https://doi.org/10.1172/jici. insight.88609.

\section{Introduction}

More than 25,000 extremely preterm infants are born annually in the United States (approximately $0.6 \%$ of all births), and this number is reported to be increasing by $7 \%$ each year (1). Although the survival rate of extremely preterm infants has improved, many survivors (25\%-50\%) develop cognitive dysfunction in later life (2-4). The reason why cognitive impairment is a common neurological outcome following brain injury in extremely preterm infants remains unknown $(5,6)$. Diffuse white matter injury (WMI) has been identified as the most common neuropathology in extremely preterm infants, rather than cystic periventricular leukomalacia, which is frequently present in the injured brains of preterm infants born later than extremely preterm infants $(5,7,8)$. Failure of oligodendrocyte maturation is thought to underlie the myelination disturbances in WMI (9-11). However, these pathological changes in the white matter are considered insufficient to completely explain the cognitive impairment that often develops in surviving extremely preterm infants. 
There is increasing interest in the role of gray matter injury as a cause of the altered brain growth and subsequent development of cognitive dysfunction in extremely preterm infants (10-12). Disturbances of maturation of various cellular components of the cortical neurons, such as the dendritic arbors and synapse formation, have been shown to be related to impaired cerebral growth in a preterm large-animal model (13). Recent studies have also suggested perturbed maturation of inhibitory interneurons, caudate projection neurons, and astrocytes by using animal models (14-16). Furthermore, as an underlying cause of the altered brain growth and subsequent development of cognitive dysfunction, brain injury in premature infants is thought to affect radial neuronal migration in the neocortex (17); however, this idea has not yet been rigorously investigated (6).

Neuronal migration is a critical step in the formation of the highly organized mammalian neocortex. Until recently, it was considered that rat E21 (the last day of pregnancy) corresponds to human gestational weeks (GWs) 15-16 and that, in the human brains, neurogenesis is completed by the end of the 16th week $(18,19)$; thus, the prevailing rat model of perinatal hypoxic-ischemic brain injury uses postnatal pups in which neurogenesis has been completed (20). However, recent findings indicate that human neocortical neurogenesis continues into the third trimester $(21,22)$, suggesting continuation of neuronal migration following neurogenesis even after $23 \mathrm{GWs}$, the gestational week at which extremely preterm infants have live births. Thus, in this study, we have investigated whether altered neuronal migration might be involved in the pathogenesis of the cognitive impairment that often develops later in extremely preterm infants with brain injury.

\section{Results}

Analyses of neocortical development in human infants. We first examined the histological features of the human neocortex by Nissl staining using a series of fetal brain sections (Figure 1A, Supplemental Figure 1A, and Supplemental Table 1; 8 brains between $19 \mathrm{GWs}$ and $38 \mathrm{GWs;} \mathrm{supplemental} \mathrm{material} \mathrm{available} \mathrm{online} \mathrm{with}$ this article; https://doi.org/10.1172/jci.insight.88609DS1). We observed numerous "cell bands" radiating from the subventricular zone (SVZ) toward the intermediate zone (IZ) until $28 \mathrm{GWs}$, with the bands becoming faint by 38 GWs (Figure 1, B and D). These "cell bands" were particularly prominent until 31 GWs in horizontally sliced tissue sections across the ventricles (Figure 1E and Supplemental Figure 1A). These "cell bands" were thought to be streams of migrating neurons (22) and were actually found to contain cells with the morphological features of migrating neurons (Figure 1F, arrowheads). In addition, at 28 and $31 \mathrm{GWs}$, but not at $38 \mathrm{GWs}$, the outermost regions of the neocortex showed cell-dense accumulations of neurons resembling the primitive cortical zone (PCZ) in the mouse and Macaca $(23,24)$ (Figure $1 \mathrm{C}$ and Supplemental Figure 1B). Since the PCZ consists of excitatory neurons that have migrated most recently to the top of the cortical plate $(23,24)$, the presence of the PCZ suggests that neurons continue to migrate into the cortical plate until around $30 \mathrm{GWs}$.

Further examination of comparison cases between 23 GWs and 26 GWs (Supplemental Table 2; 23 GWs: $n=4,24$ GWs: $n=1,26$ GWs: $n=2$ ) showed that the ventricular zone (VZ) and inner SVZ (ISVZ) of the human neocortex are composed of layered neural progenitors, suggestive of ongoing neurogenesis (Figure 2, A and B, and Supplemental Table 2). Immunostaining with Musashi1 and nestin antibodies at 26 GWs (Figure 2, B and C) revealed that the cells in the VZ, ISVZ, and "cell bands" in the outer SVZ (OSVZ) expressed both of these neural progenitor/precursor markers. In addition, vimentin immunolabeling identified streams of vimentin-positive radial processes and cell bodies (Figure 2, A, arrows, and C and D), indicating that at 26 GWs, the neocortex has an infrastructure that is capable of supporting the migration of the neurons generated. In addition, examination of H\&E-stained sections at higher magnifications revealed the presence of cells with long leading processes, a characteristic feature of migrating neurons, throughout the neocortex (Figure 2D). Importantly, they expressed one of the early neuronal markers, HuB (25) (Figure 2D).

Immunolabeling for doublecortin (DCX, a neuronal marker; ref. 26) also confirmed the existence of migrating cells that expressed neuronal markers in the human neocortex at 23 and 25 GWs (Figure 2E; Supplemental Figure 2, A and B; and Supplemental Table 1; 23 GWs: $n=3,25$ GWs: $n=2$ ). These cells were assumed to be superficial layer neurons, since double immunolabeling for DCX and CUX2 (a marker predominantly expressed in the superficial layer neurons; ref. 27) in the human neocortex at 25 GWs revealed that the DCX-positive cells were also moderately positive for CUX2 (Figure 2F, arrows). These DCX-positive cells were also positive for BRN2 (another marker predominantly expressed in the superficial layer neurons; ref. 28) (Figure 2G). Moreover, cells moderately positive for CUX1 (another 
A
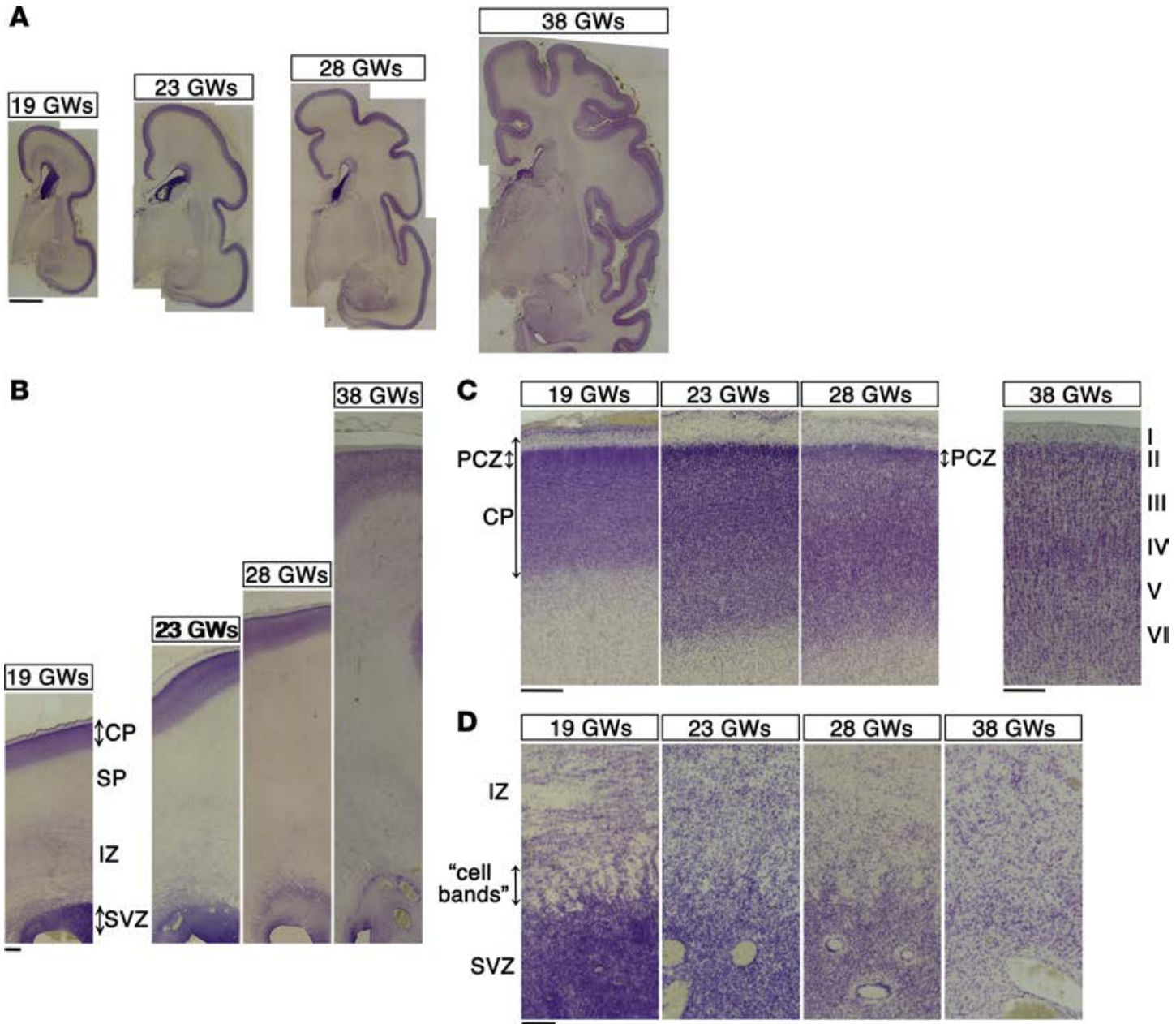

$\mathbf{E}$
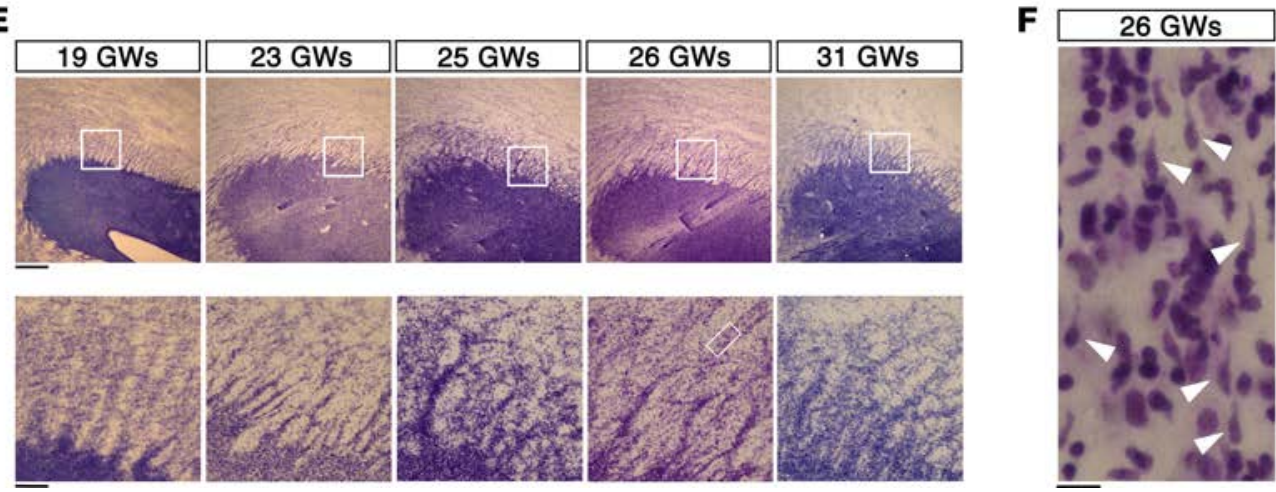

Figure 1. Histological features of the developing human neocortex. (A) Coronal sections from the human neocortex at 19, 23, 28, and $38 \mathrm{CWs}$ were stained with Cresyl violet (Nissl staining). Scale bar: $5 \mathrm{~mm}$. (B) High-magnification images of the dorsolateral neocortex in A. Scale bar: $1 \mathrm{~mm}$. SP, subplate.(C) High-magnification images of the forming cortical plate (CP) in B. Note that the outermost regions of the neocortex at 19,23 , and 28 $\mathrm{CWs}$, but not at $38 \mathrm{CWs}$, showed cell-dense accumulation of neurons. This region is thought to correspond to the primitive cortical zone (PCZ). Scale bar: $500 \mu \mathrm{m}$. (D) High-magnification images of the subventricular zone (SVZ) in B. Numerous "cell bands" radiating from the SVZ toward the intermediate zone (IZ) were recognized until $28 \mathrm{GWs}$ but became faint by $38 \mathrm{GWs}$. Scale bar: $200 \mu \mathrm{m}$. (E) The "cell bands" were particularly prominent in horizontally sliced sections of the neocortex at 19 to $31 \mathrm{CWs}$ (lower-magnification images are shown in Supplemental Figure 1). Boxed areas in the top panels are shown at high magnification in the bottom panels. Scale bar: $500 \mu \mathrm{m}$ (top); $100 \mu \mathrm{m}$ (bottom). (F) A further magnification of the boxed area in the bottom panel in E (26 GWs). The "cell bands" contained plenty of cells with the morphological features of migrating neurons (white arrowheads). Seven focal planes were merged to visualize the shapes of the entire cells. Scale bar: $10 \mu \mathrm{m}$. 


\section{A}
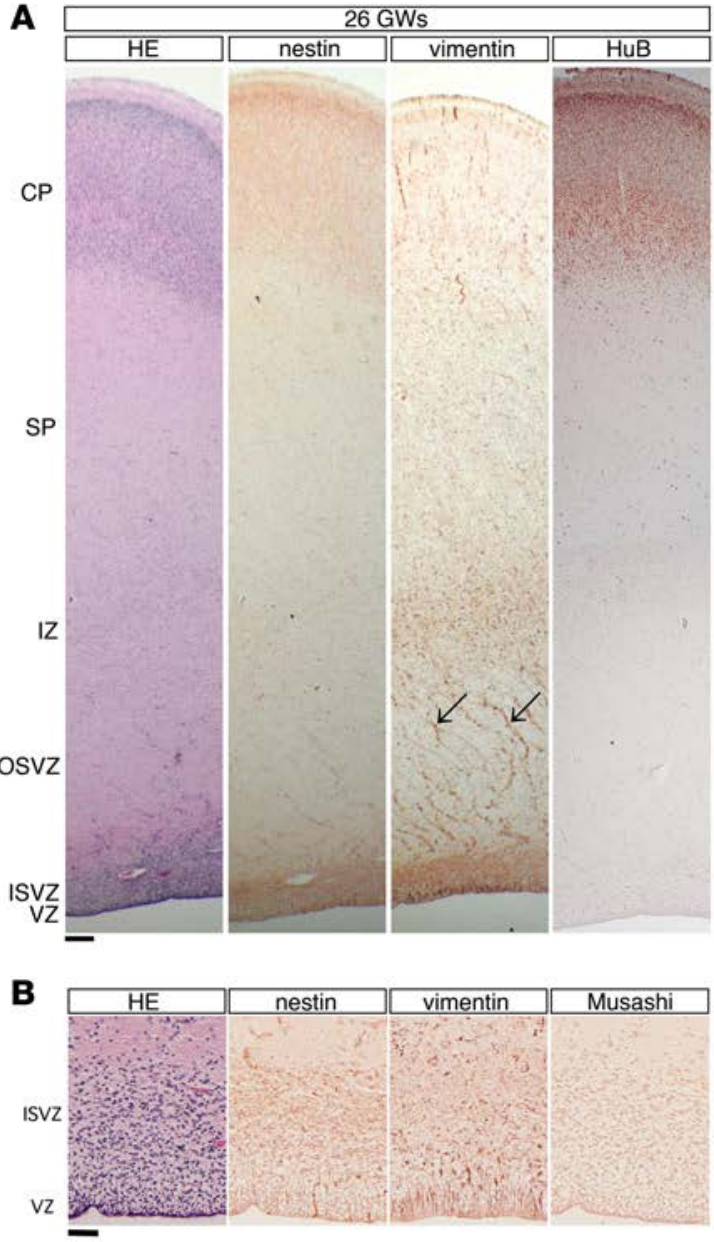
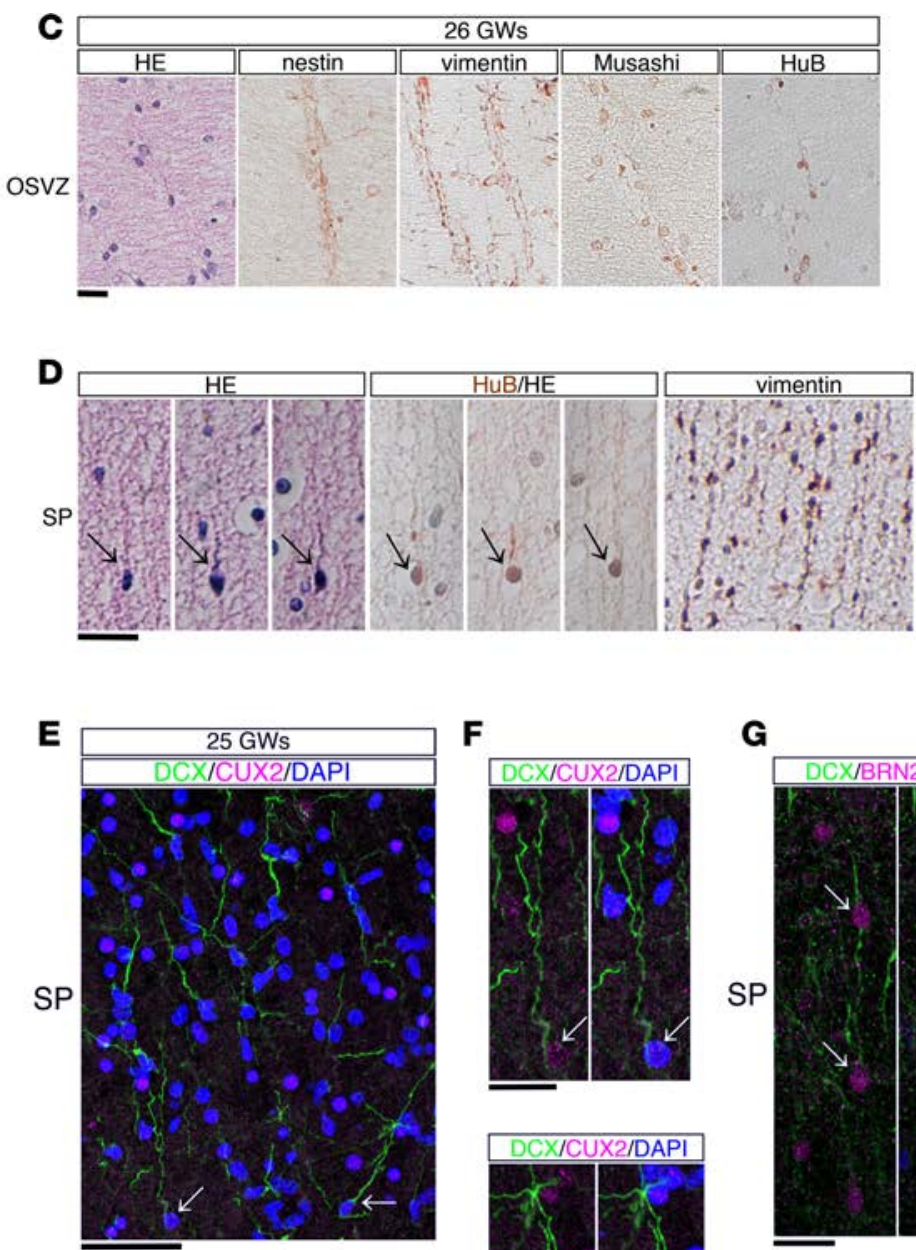

$\mathbf{F}$
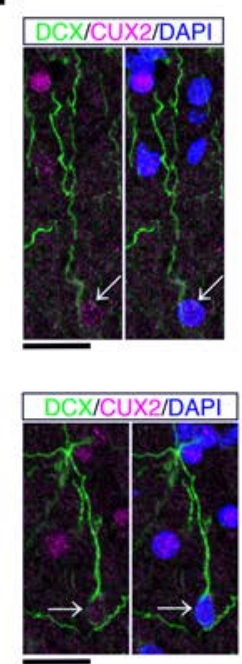

G

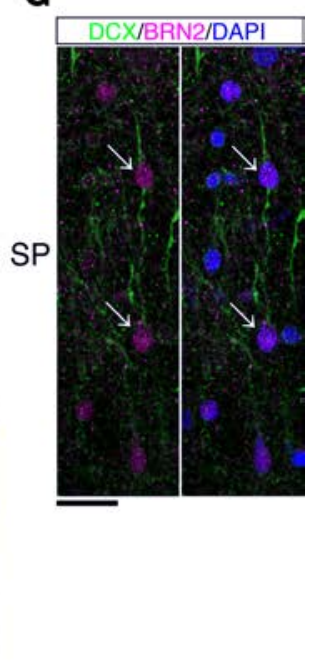

Figure 2. Analyses of neocortical development in human infants. (A) Sections from the human neocortex at $26 \mathrm{CWs}$ were stained with H\&E (HE) and immunostained with anti-nestin antibody, anti-vimentin antibody, and anti-HuB antibody. The vimentin-positive fibers in the outer subventricular zone (OSVZ) were in bundles (arrows). CP, cortical plate; SP, subplate; IZ, intermediate zone; ISVZ, inner subventricular zone; VZ, ventricular zone. Scale bar: $200 \mu \mathrm{m}$. (B) High-magnification images of images in A showing the ISVZ/VZ immunostained with anti-nestin antibody, anti-vimentin antibody, and anti-Musashi1 antibody. Scale bar: $50 \mu \mathrm{m}$. (C) High-magnification images of the OSVZ. Scale bar: $10 \mu \mathrm{m}$. (D) High-magnification images of the SP. The vimentin-positive fibers in the SP were separated and contained varicosities. Cells with leading processes were present (arrows). Scale bar: $10 \mu \mathrm{m}$. (E and $\mathbf{F}$ ) Sections from the human occipital neocortex at $25 \mathrm{CW}$ s were stained with anti-DCX (green) and CUX2 (magenta) antibodies. High-magnification images of the cells in $\mathbf{E}$ (indicated by arrows) are shown in $\mathbf{F}$ (indicated by arrows). Scale bar: $50 \mu \mathrm{m}(\mathbf{E}) ; 20 \mu \mathrm{m}$ (F). (C) Sections from the human occipital neocortex at $25 \mathrm{GWs}$ were stained with anti-DCX (green) and BRN2 (magenta) antibodies. The cells with DCX-positive processes were also positive for BRN2 (indicated by arrows). Scale bar: $20 \mu \mathrm{m}$.

superficial layer neuron marker, also known as CUTL1; ref. 27) were diffusely distributed throughout the neocortex, including the IZ and subplate (SP), especially at 23 GWs (29) (Supplemental Figure 2, C and $\mathrm{D}$, arrows). We considered that these cells moderately positive for CUX1 were still migrating or had just completed migration, since they were found in abundance, especially at the top of the cortical plate (Supplemental Figure 2E, blue arrows). Based on the above observations, we concluded that neuronal migration continues to occur even after $23 \mathrm{GWs}$ in human fetuses.

Analyses of human neocortices from extremely preterm infants. To determine whether neuronal migration is affected in extremely preterm infants with brain injury, we first examined the autopsy brains of 41 extremely preterm infants with a pathological diagnosis of WMI (mean gestational age at birth: $25.0 \pm 1.4 \mathrm{GWs}$, range: 23-27 GWs; Supplemental Table 3). The morphologic features of WMI of extremely preterm infants $(5,30-32)$ were identified as diffuse lesions in all cases (Supplemental Table 4). Cyst formation in the white 

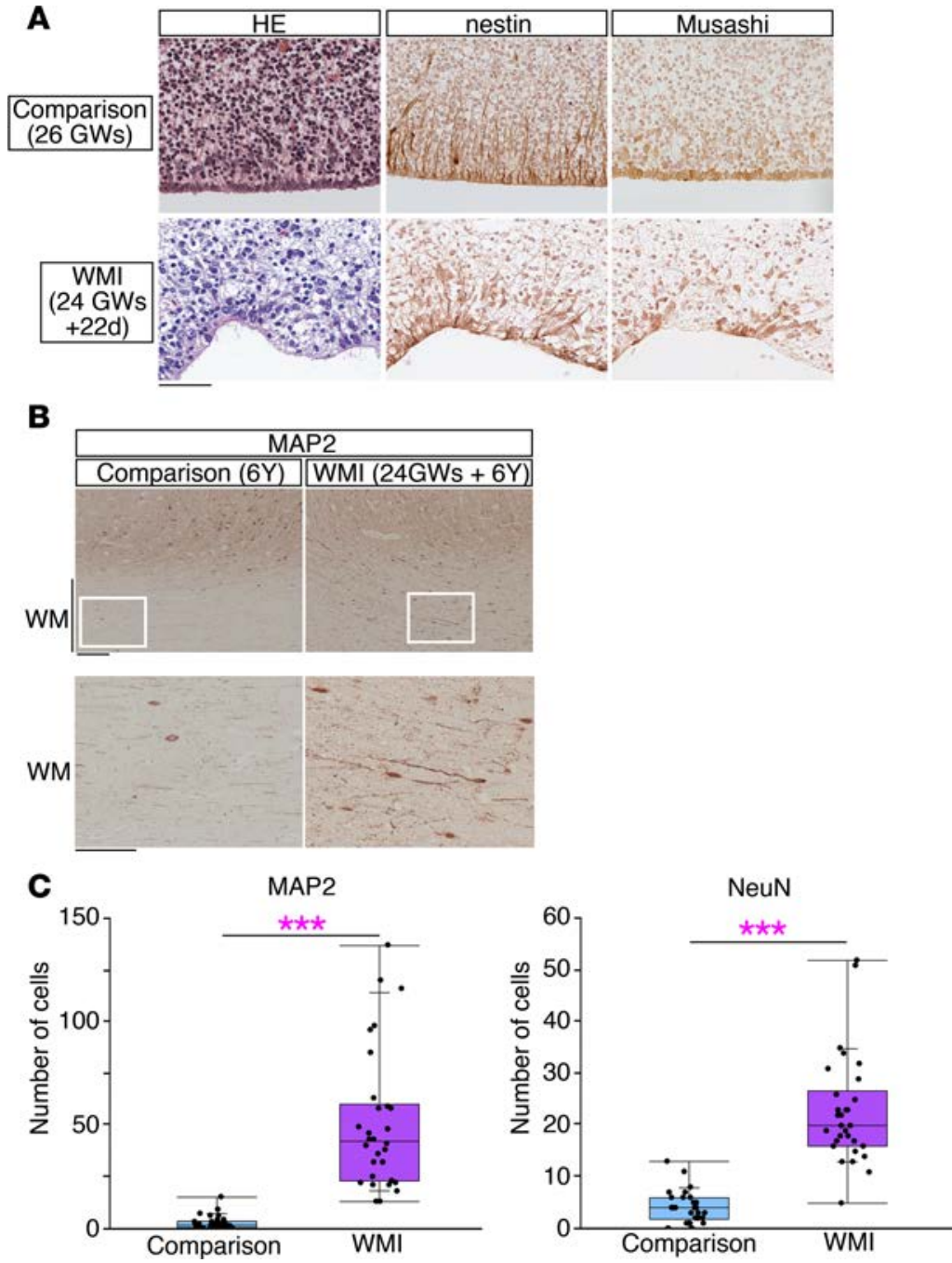

Figure 3. Histological analyses of neocortices from extremely preterm infants. (A) Histological appearance of the subventricular zone/ventricular zone of the neocortices of a comparison case (26 GWs) and an extremely preterm infant with white matter injury (WMI) (24 GWs at birth, survived 22 days). Sections were stained with H\&E (HE) and immunostained with anti-nestin antibody and anti-Musashi antibody. Scale bar: $50 \mu \mathrm{m}$. (B) Sections of neocortices of a comparison case (6 years old) and an extremely preterm infant with WMI (24 CWs at birth, survived 6 years) immunostained for MAP2. High-magnification images of boxed areas of white matter (WM) are shown. Scale bar: $50 \mu \mathrm{m}$. (C) MAP2-positive cells and NeuN-positive cells in the white matter were quantified by counting the number of these cells in 10 randomly acquired digitized high-power field images (original magnification, $\times 20)$. The numbers of MAP2-positive cells and NeuN-positive cells per field are shown ( $n=30$ fields from 3 different brains, respectively). ${ }^{* * *} P<0.001$, Welch's $t$ test. Each point represents an individual field. Box-and-whisker plots were used to graphically represent the median (line within box), upper and lower quartiles (bounds of box), and maximum and minimum values (top and bottom bars).

matter, a pathologic landmark of classic periventricular leukomalacia, was observed in only 4 cases (Supplemental Table 4 and Supplemental Figure 3). The cellular organization in the VZ/SVZ/ependymal epithelium was disrupted to various degrees (Supplemental Table 5 ), which frequently resulted in an irregular ventricular surface and a discontinuous pattern of immunostaining for nestin and Musashi1 (Figure 3A and Supplemental Figure $4 \mathrm{~A}$, bottom). The degree of disruption of the $\mathrm{VZ/SVZ/ependymal} \mathrm{epithelium} \mathrm{tended} \mathrm{to} \mathrm{be} \mathrm{more}$ extensive in the long-term survivors ("long-term cases" with WMI who survived 21 months to 6 years) (Supplemental Figure 4C) and was not significantly different between survivors with and without intraventricular hemorrhage (Supplemental Figure 4, D and E). The disruption of the VZ/SVZ/ependymal epithelium suggested that preterm brain injury can affect the neural progenitor/precursor cells that support neuronal migration.

To identify possible consequences of the preterm brain injury for subsequent neural development, we examined the white matter and cortices of 3 long-term cases and 3 comparison cases for the postmitotic neuronal markers NeuN and MAP2 (Figure 3B). There were significantly more MAP2- and NeuN-positive neurons in the white matter in the long-term cases than in the comparison cases (Figure 3C). The MAP2-positive cells seemed to be diffusely distributed in the white matter of the brain in the long-term cases, with slight local variation (Supplemental Figure 5 and 6). The GFAP-positive cells were also distributed in the white matter in the long-term cases, with local variation (Supplemental Figures 5 and 6), as has been reported for premature brains at later stages (9), but the number of MAP2-positive neurons in the white matter did not seem to be correlated with the number of GFAP-positive cells (Supplemental Figure 6C).

Effect of brain injury on neurodevelopment in the mouse neocortex. In order to determine whether preterm brain injury affects neuronal migration and results in an increased number of white matter neurons, we sought to create a suitable mouse model. For comparing the pattern of neuronal migration, we stained the developing human and mouse brains with antibodies against human BRN2 and mouse homologue Brn2, respectively. In the developing human neocortex, BRN2-positive neurons were abundantly distributed in the SP or IZ, in addition to the upper cortical plate, at $23 \mathrm{GWs}$ and $25 \mathrm{GWs}$ (Figure 4, A-C, and Supplemental Figure 7; $23 \mathrm{GWs:}$ $n=3,25$ GWs: $n=2$ ). The ratio of BRN2-positive neurons in the cortical plate of the human developing brain to that of total BRN2-positive neurons in the neocortical wall at 23-25 GWs was $0.2-0.3$, which seemed to correspond to the proportion of Brn2-positive neurons in the mouse cortical plate at E15.5-E16.5 (Figure 4, D-F). 
A

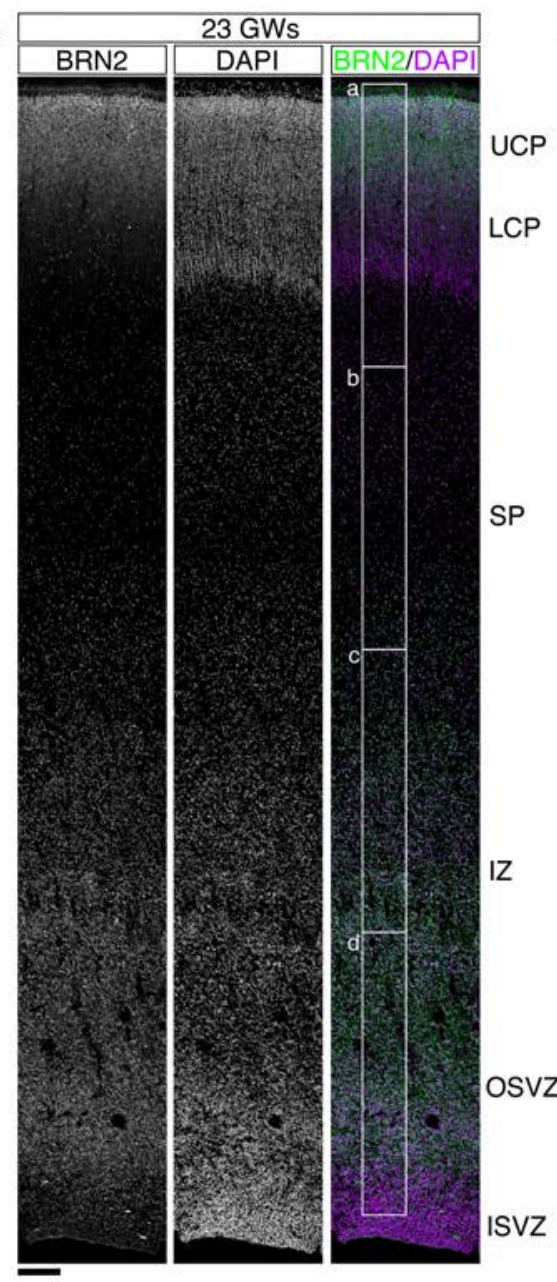

B

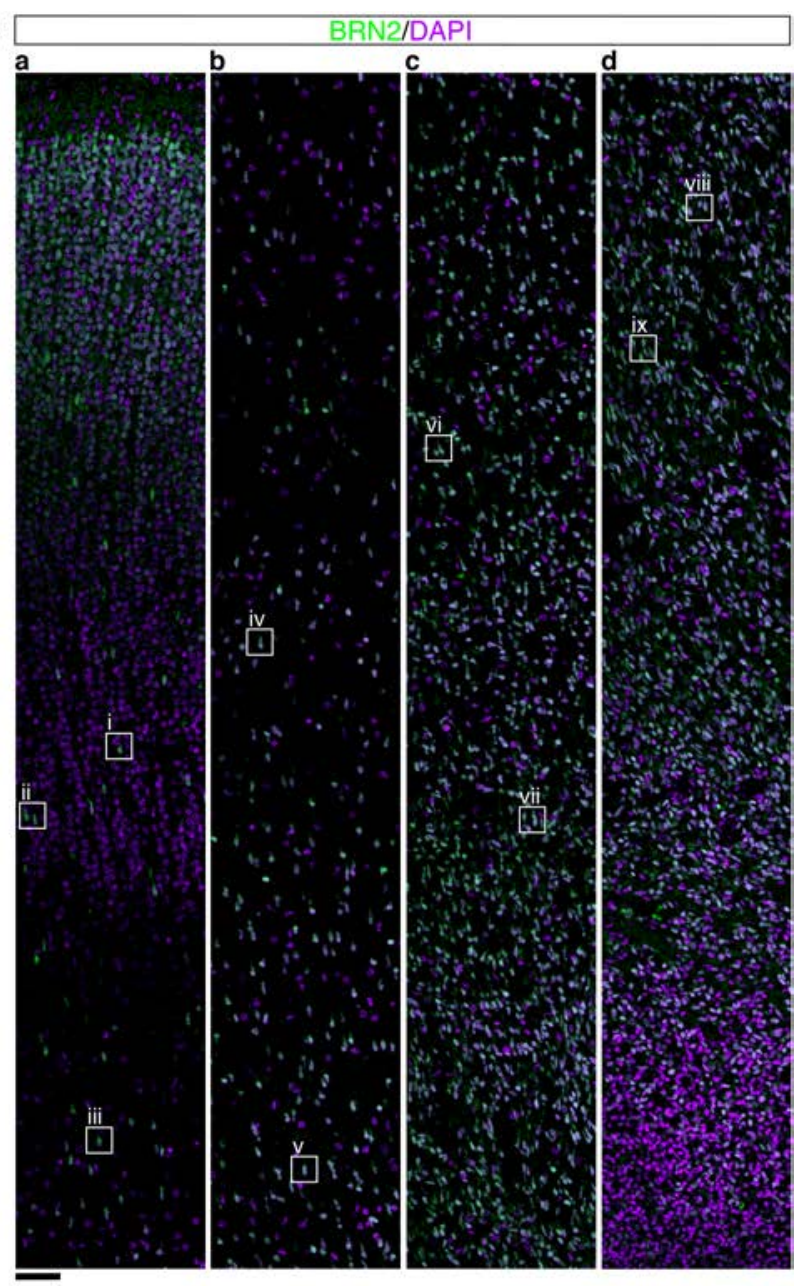

C

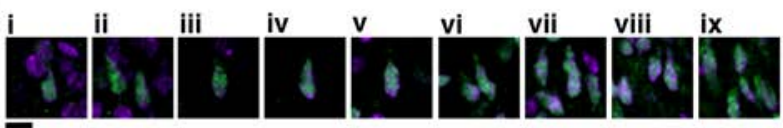

D

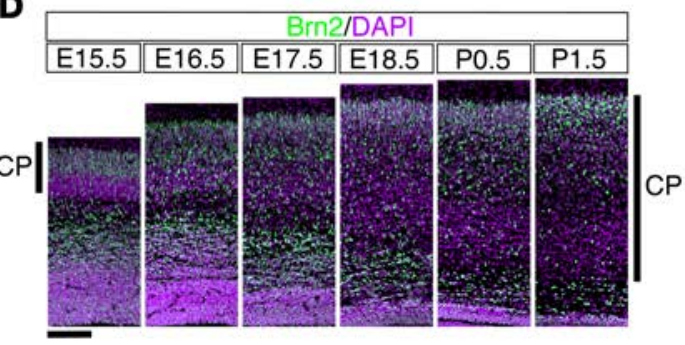

E

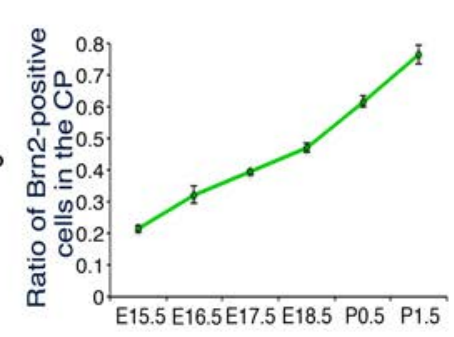

$\mathbf{F}$

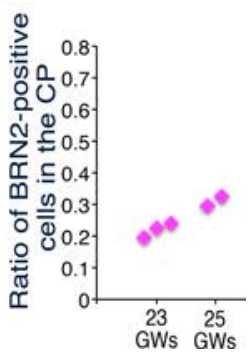

Figure 4. BRN2-positive cells were abundantly distributed in the subplate and intermediate zone of the human neocortex at $23 \mathrm{GWs.} \mathrm{(A)} \mathrm{Sections} \mathrm{from}$ the human occipital neocortex at $23 \mathrm{CWs}$ were stained with anti-BRN2 antibody (left; gray scale, right; green) and counterstained with DAPI (middle; gray scale, right; purple). (B) High-magnification images of the boxed areas in A (labeled a-d) are shown. The BRN2-positive cells accumulated at the top of the cortical plate (CP) and were diffusely distributed throughout the neocortex, including the subplate (SP) and intermediate zone (IZ). (C) High-magnification images of the squares in B (labeled i-ix) are shown. Scale bar: $200 \mu \mathrm{m}$ (A); $50 \mu \mathrm{m}$ (B); $10 \mu \mathrm{m}$ (C). (D) Sections from the developing mouse neocortex from E15.5 to P1.5 were stained with an anti-Brn2 (green) antibody and counterstained with DAPI (purple). Scale bar: $200 \mu \mathrm{m}$. (E) The ratio of the number of Brn2-positive cells in the CP to the total number of Brn2-positive cells throughout the mouse neocortical wall was calculated (mean \pm SEM; $n=4$, respectively). (F) The ratio of the number of BRN2-positive cells in the CP to the total number of BRN2-positive cells throughout the human neocortical wall (23 CWs: $n=3,25 \mathrm{CWs}: n=2$ ) was calculated and plotted. 
A
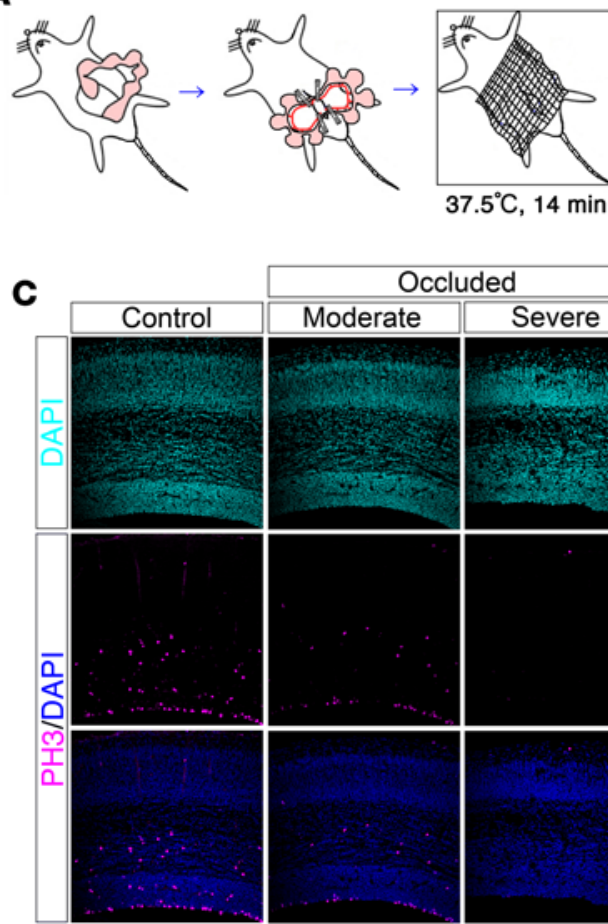

D

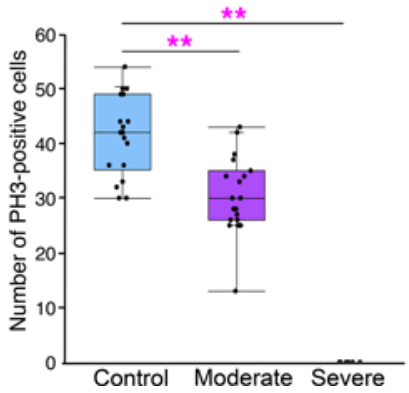

B

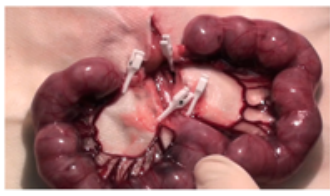

E

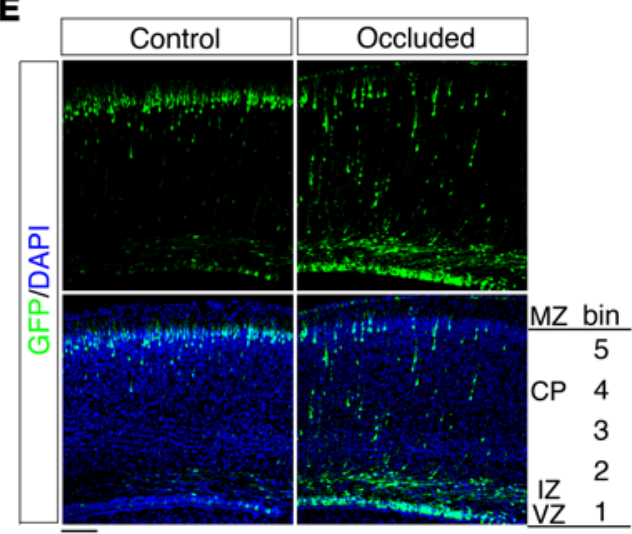

$\mathbf{F}$

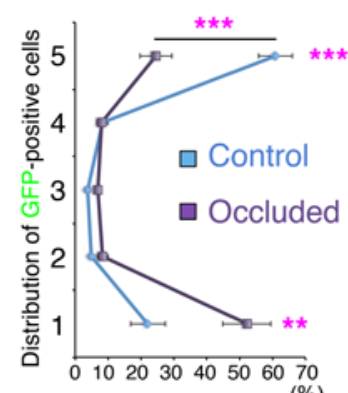

G

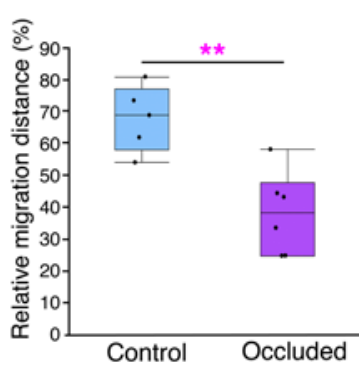

Figure 5. Effect of brain injury on neurodevelopment in the mouse neocortex. (A) Schematic representation of the procedure for producing the mouse model of brain injury. (B) Representative photograph of maternal uterine arteries occluded with 4 disposable vascular clips. (C) Brains of control embryos (left) and occluded embryos (middle and right) 12 hours after occlusion. Sections were immunostained with anti-PH3 antibody (counterstained with DAPI). SVZ, subventricular zone; VZ, ventricular zone. Scale bar: $100 \mu \mathrm{m}$. (D) The numbers of PH3-positive cells in the VZ and SVZ are shown (control: $n=18$, moderate: $n=19$, severe: $n=6$ ). A Kruskal-Wallis test followed by Dunnett's post-hoc test was used. ${ }^{* *} P<0.01$. (E) After transfection of GFP expression plasmid at E15.0, a sham operation (Control) or maternal uterine artery occlusion (Occluded) was performed at E16.5. Brains were analyzed at P1 (counterstained with DAPI [blue]). MZ, marginal zone; CP, cortical plate. Scale bar: $100 \mu \mathrm{m}$. (F) Cell distribution was evaluated by bin analysis. Data obtained in 5 control brains and 6 occluded brains (mean $\pm \mathrm{SEM}$ ) are shown. ${ }^{* *} P<0.01,{ }^{* *} P<0.001$, repeated-measures ANOVA followed by Bonferroni post-hoc test. (C) Relative migration distances (\%) from the ventricle. Average relative migration distances in 5 control brains and 6 occluded brains are shown. ${ }^{* *} P$ $<0.01$, Student's $t$ test. ( $\mathbf{D}$ and $\mathbf{G}$ ) Each point represents an individual mouse. Box-and-whisker plots were used to graphically represent the median (line within box), upper and lower quartiles (bounds of box), and maximum and minimum values (top and bottom bars).

Based on the above comparison, we transiently occluded the maternal uterine arteries with clips at E16.5 (occluded group) (Figure 5, A and B). We carried out arterial blood gas analysis of blood samples obtained from the occluded maternal uterine arteries and assumed that adequate hypoxia had been induced by this method (Supplemental Table 6). In addition, the transient maternal uterine artery occlusion at E16.5 caused a significant decrease in litter size at birth (occluded group: $3.5 \pm 0.4$ pups [ $n=83$ ], sham operation group: $10.3 \pm 0.7$ pups $[n=16], P=1.13 \mathrm{e}-09$, Student's $t$ test), which was evidence of substantial damage to the embryos.

When we attempted to harvest the brains of the embryos 12 hours after the occlusion, we found that $5.9 \%$ (6 of 101) of the "occluded" brains (this term is used to indicate the brains of embryos/mice that have experienced the transient maternal uterine artery occlusion at E16.5 in the rest of this study) were too damaged to be harvested. When we sectioned and analyzed the occluded brains, approximately 

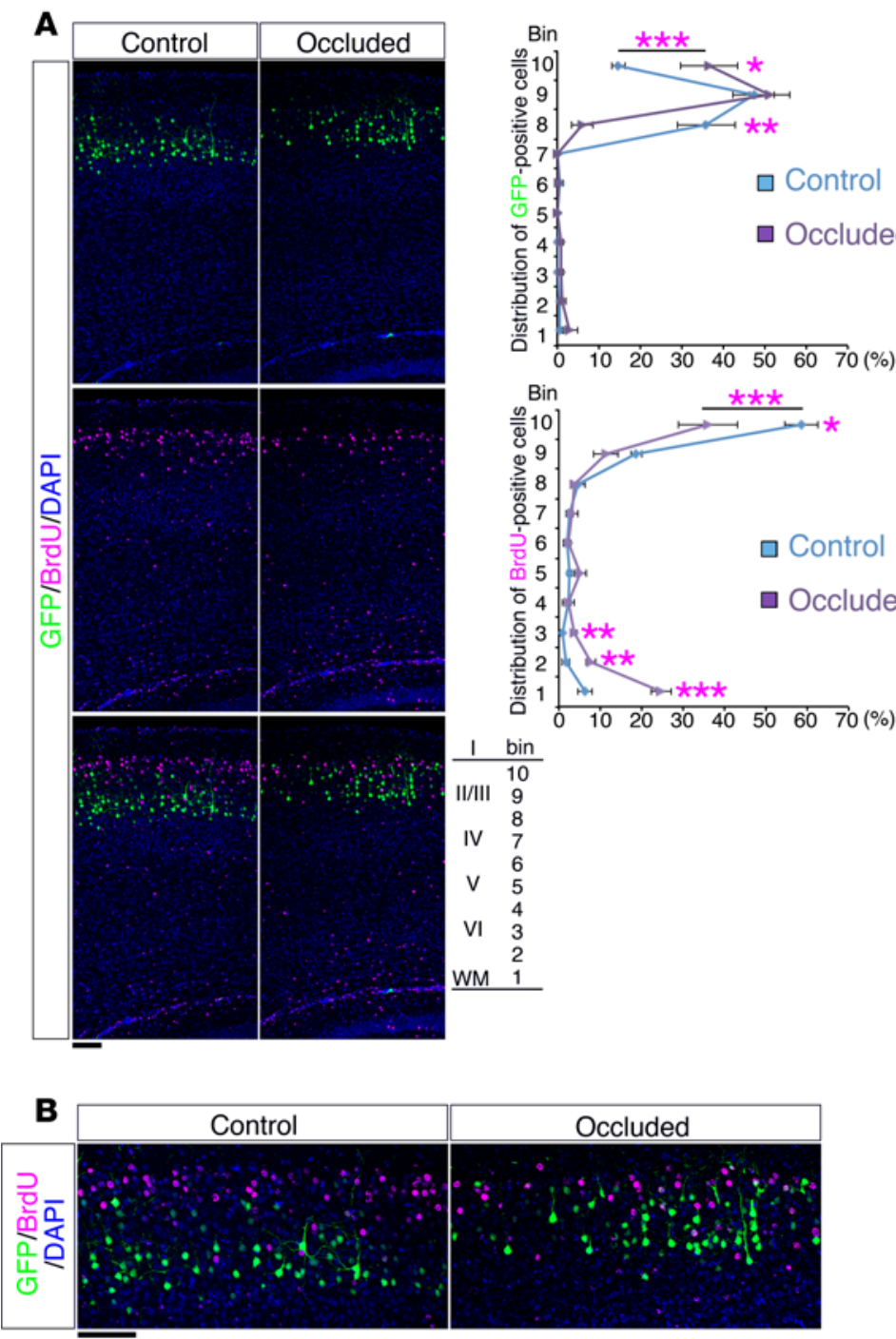

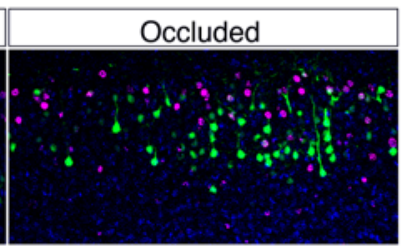

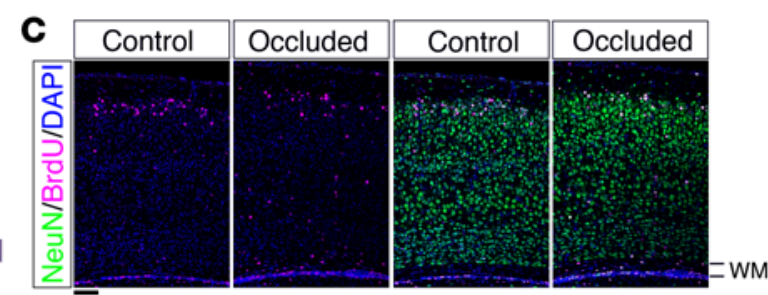

D
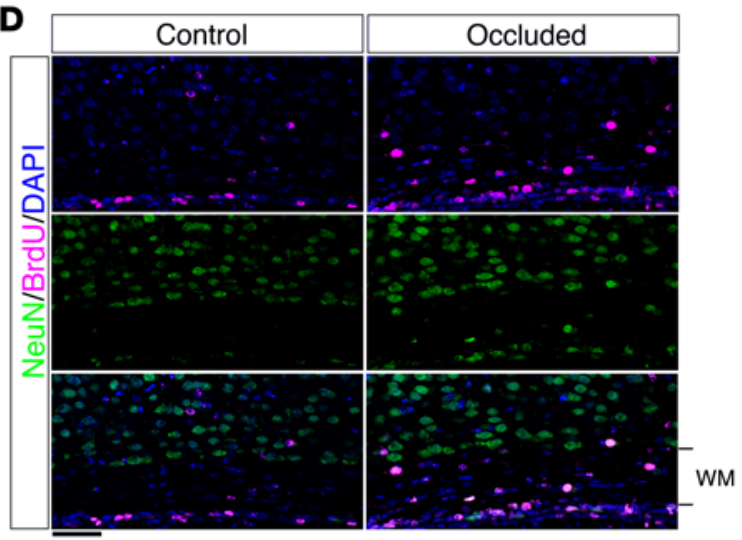

$\mathbf{E}$

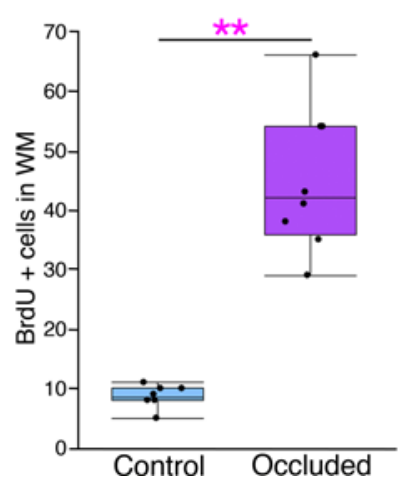

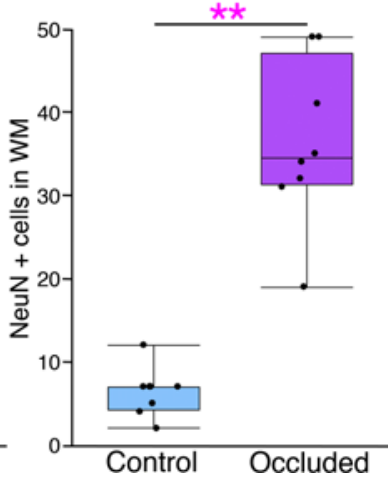

Figure 6. The mouse model showed an altered neuronal alignment. (A) After transfection of GFP plasmid at E15.0, a sham operation (Control) or maternal uterine artery occlusion (Occluded) was performed at E16.5 and followed by BrdU injection at E17.0. Brains were analyzed at P10. Sections were immunostained with anti-BrdU antibody (magenta). Right panels show the bin analysis of the distribution of GFP-positive cells and BrdU-positive cells (mean \pm SEM; $n=4$, respectively). ${ }^{*} P<0.05,{ }^{* *} P<0.01$, ${ }^{* * *} P<0.001$, repeated-measures ANOVA followed by Bonferroni post-hoc test. Scale bar: 100 $\mu \mathrm{m}$. (B) High-magnification images of the superficial areas of the neocortices in A. Scale bar: $100 \mu \mathrm{m}$. (C) A sham operation or maternal uterine artery occlusion was performed at E16.5 and followed by BrdU injection at E17.0. Brains were analyzed at P10. Sections were immunostained with anti-NeuN antibody (green) and anti-BrdU antibody (magenta). Scale bar: $100 \mu \mathrm{m}$. (D) High-magnification images of the white matter (WM) in C. Scale bar: $50 \mu \mathrm{m}$. (E) The number of BrdU-positive cells (left) and NeuN-positive cells (right) in the WM ( $n=8$, respectively) are shown. ${ }^{* *} P<0.001$, Welch's $t$ test. Each point represents an individual mouse. Box-and-whisker plots were used to graphically represent the median (line within box), upper and lower quartiles (bounds of box), and maximum and minimum values (top and bottom bars).

three-fourths ( $76.9 \%, 30$ of the 39 brains, Figure 5C and Supplemental Figure 8, moderately damaged brains) showed an almost normal structure of the neocortex, whereas, in the remaining approximately one-fourth of the brains (23.1\%, 9 of 39 brains) (Figure 5C and Supplemental Figure 8, severely damaged brains), the neocortical cytoarchitecture was severely altered, resulting in a disorganized cortical plate with a cobblestone-like pattern. Immunostaining of brain sections with an anti-active caspase-3 antibody to visualize apoptosis revealed active caspase-3-positive cells in several regions of the severely damaged brains, whereas few active caspase-3-positive cells were in the moderately damaged brains (Supplemental Figure 8). To evaluate neurogenesis, we stained brains with an anti-phospho histone 3 (anti-PH3) antibody to identify cell division, and the results showed that few cells in the severely damaged brains were positive (Figure 5C). There were also significantly fewer PH3-positive cells in the VZ 
and SVZ in the moderately damaged brains, indicating that progenitor/precursor proliferation is susceptible in our model (Figure 5, C and D).

To determine whether neuronal migration was altered by the maternal uterine artery occlusion, we transfected a GFP plasmid into the neocortical ventricular surface at E15.0 and at E16.5 occluded the maternal uterine arteries (occluded group) or performed a sham operation (control group). Staining of the occluded brains with DAPI at P1 revealed almost normal neocortical structures (Figure 5E, bottom right). The severely altered cortical plate, with a cobblestone-like pattern that was observed in the severely damaged brains, was not observed in the born pups, suggesting that these pups were embryonic survivors with moderately damaged brains. Considering the above-mentioned significant decrease of the litter size at birth, it is assumed that embryos with severely damaged brains with a disorganized cortical plate showing a cobblestone-like pattern failed to be born and/or to survive. The majority of the GFP-positive cells in the control brains at P1 had reached the top of the cortical plate (Figure 5E, left), but a large number of GFPpositive cells in the occluded brains were still in the process of migration (Figure 5E, right, and Figure 5F). The distance they had migrated was significantly shorter in the occluded brains (Figure 5G), indicating the presence of a migration defect. A similar migration defect was observed in the medial prefrontal cortex (mPFC) (Supplemental Figure 9).

To identify the changes in the expression level of the genes that might affect neuronal migration in the occluded mouse brains, we extracted the total RNAs from the control and occluded neocortices and performed microarray analyses (NCBI GEO database, the accession number is GSE89998). We obtained distinct gene expression profiles in the two groups (Supplemental Table 7), and ingenuity pathway analysis revealed "Inflammatory Disease, Inflammatory Response, Neurological Disease" as the top associated network function (Supplemental Figure 10), indicating that inflammation was one of critical contributors to brain injury in our mouse model.

The mouse model showed an altered neuronal alignment. To identify the consequences of the migration defect, we analyzed the final distribution of the GFP-labeled cells. After transfecting a GFP plasmid at E15.0, we performed a maternal uterine artery occlusion or sham operation at E16.5 and injected BrdU at E17.0. Analysis of the control brains at P10 showed that the BrdU-positive cells were arranged at the top of the cortical plate and located more superficially than the GFP-positive cells, reflecting the "insideout" pattern of neuronal alignment (Figure 6, A and B). In contrast, although some of the BrdU-positive cells were positioned at the top of the cortical plate (bin 10) in the occluded brains, their distributions were intermingled with the distributions of the GFP-positive cells (Figure 6, A and B), indicating disruption of the "inside-out" neuronal alignment. The same defect was observed in both rostral and caudal neocortical areas (Supplemental Figure 11), suggesting that the disruption of neuronal alignment had occurred throughout the neocortex.

Importantly, a certain proportion of the BrdU-positive cells in the occluded brains remained in the white matter (Figure 6C), which is reminiscent of the findings in human extremely preterm infants (Figure 3, B and C). Some of the BrdU-positive cells that remained in the white matter of the occluded brains were also positive for the neuronal marker NeuN (Figure 6D), and significantly higher proportions of both of the BrdU-positive cells and the NeuN-positive cells were distributed in the white matter of the occluded brains compared with the white matter of control brains (Figure 6E).

Defective neuronal alignment was accompanied by impaired axonal branching. To examine whether disrupted neuronal alignment affects axonal connectivity, we visualized the axons of the GFP-positive cells (Figure 7A). When a GFP plasmid was transfected at E15.0, the GFP-positive cells located in layer II/III extended their axons through the corpus callosum toward the contralateral hemisphere at P10. In the control brains, the GFP-labeled axons branched extensively in the superficial layers of the contralateral hemisphere (Figure 7A). However, in the occluded brains, extensive axonal branching was observed in the deeper layers (layers V and VI, Figure 7A). We analyzed the GFP intensity in each layer to quantify the amount of axonal branching. The GFP intensity was significantly reduced in layers I and II/III and increased in layer $\mathrm{V}$ in the occluded brains as compared with the control brains (Figure 7B), indicating that the normal corticocortical axonal wiring was altered in the occluded brains. Similar changes in axonal branching were also observed in the mPFCs of the occluded mice (Supplemental Figure 12). In the occluded brains, axons from the contralateral hemisphere seemed to make branches around ectopically distributed BrdU-positive cells in the deeper layers of the neocortex (Figure 7C, arrows), suggesting that the ectopic distribution of neurons may underlie the alterations in axonal branching. 

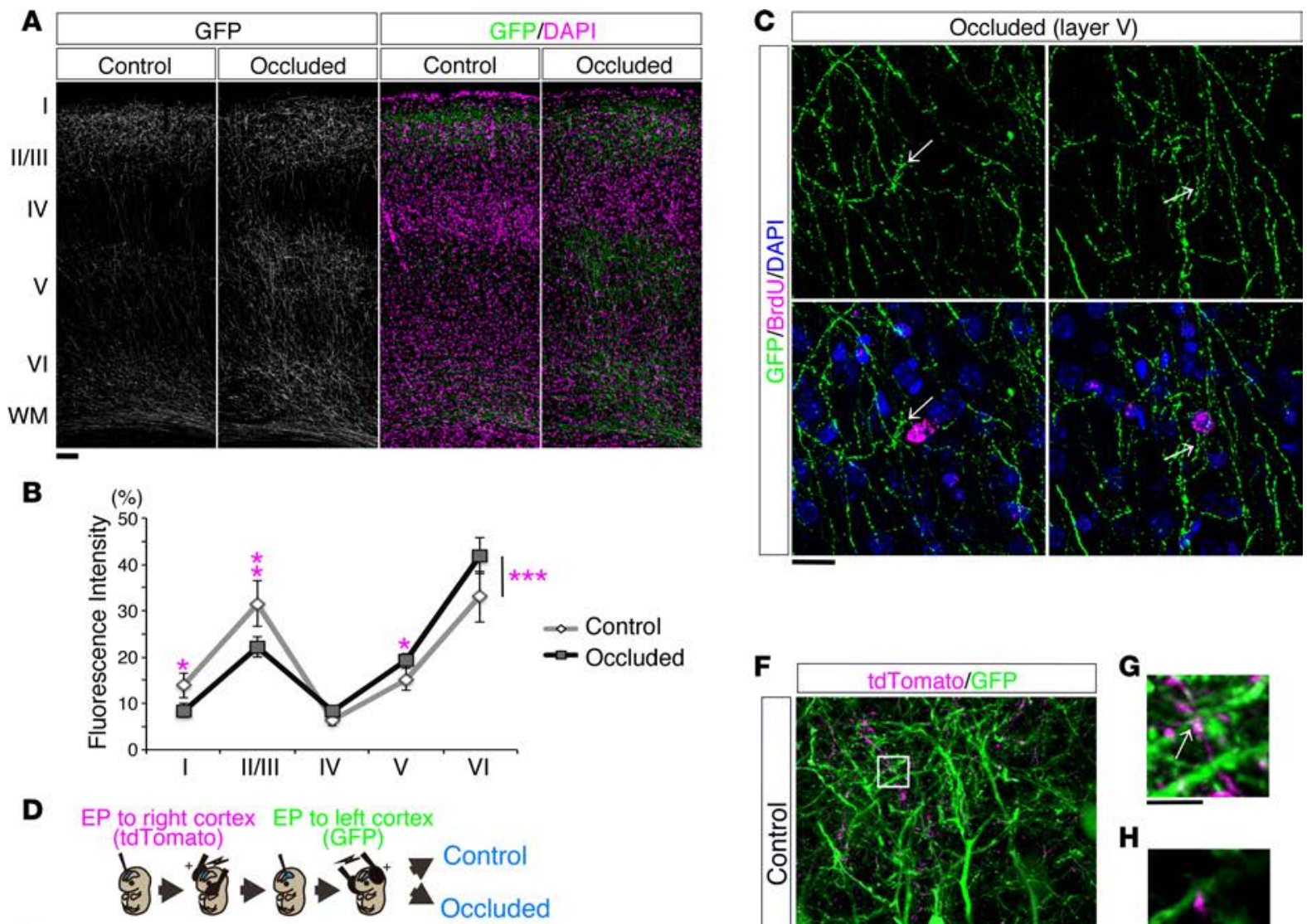

$\mathbf{E}$
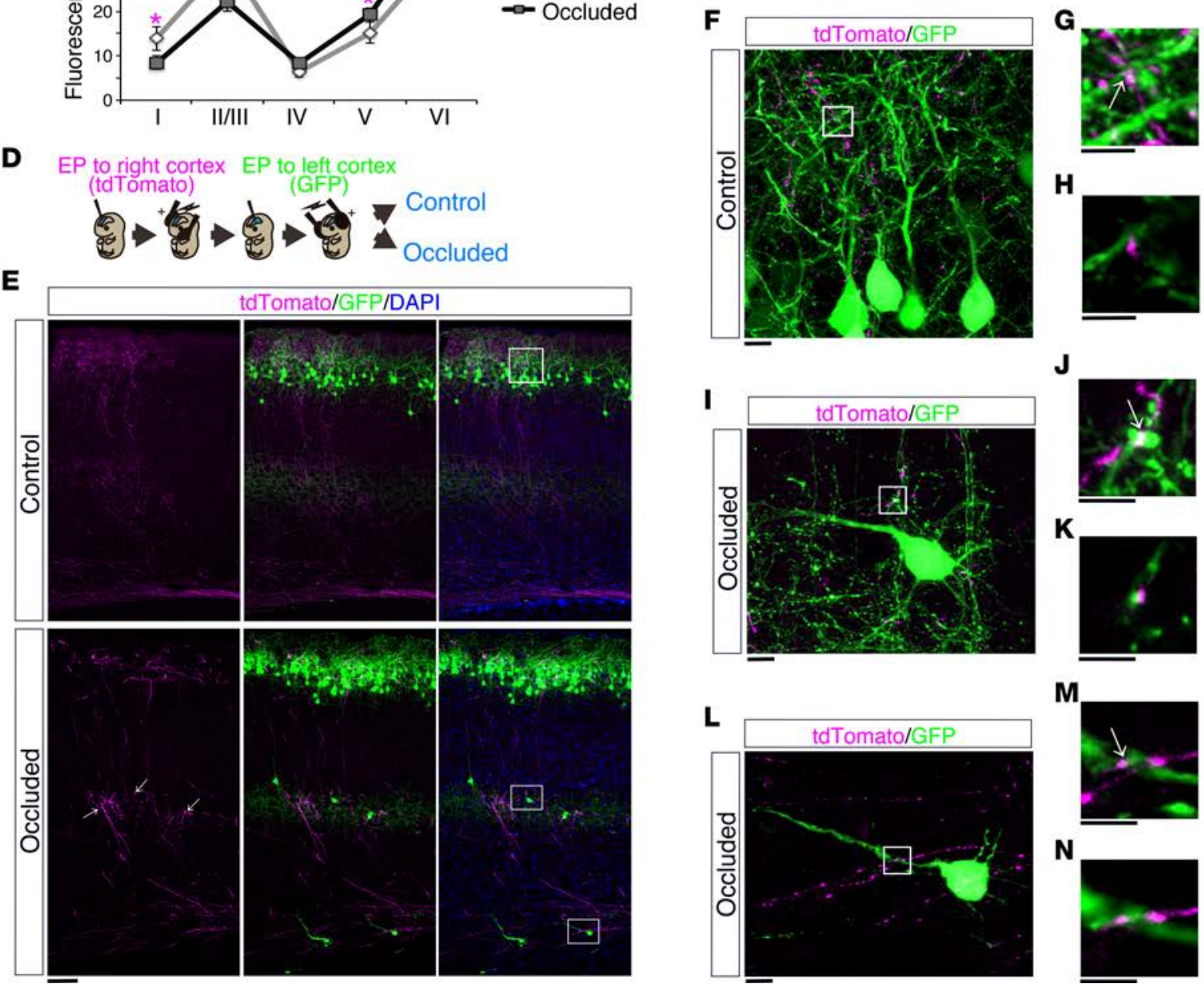

H.
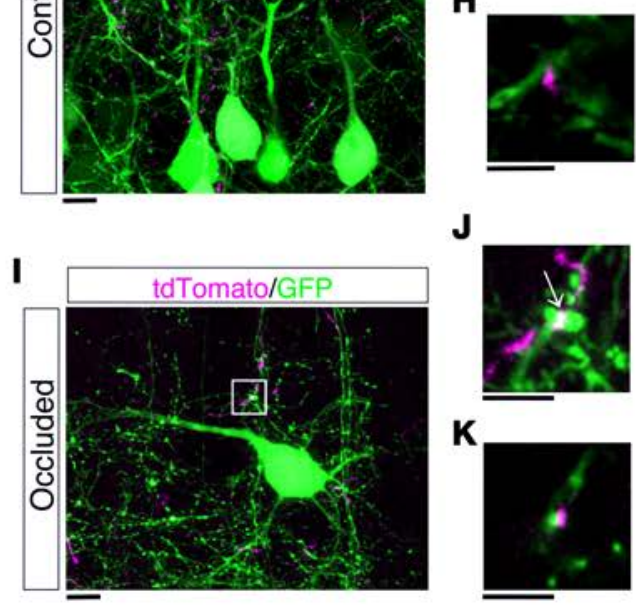

J
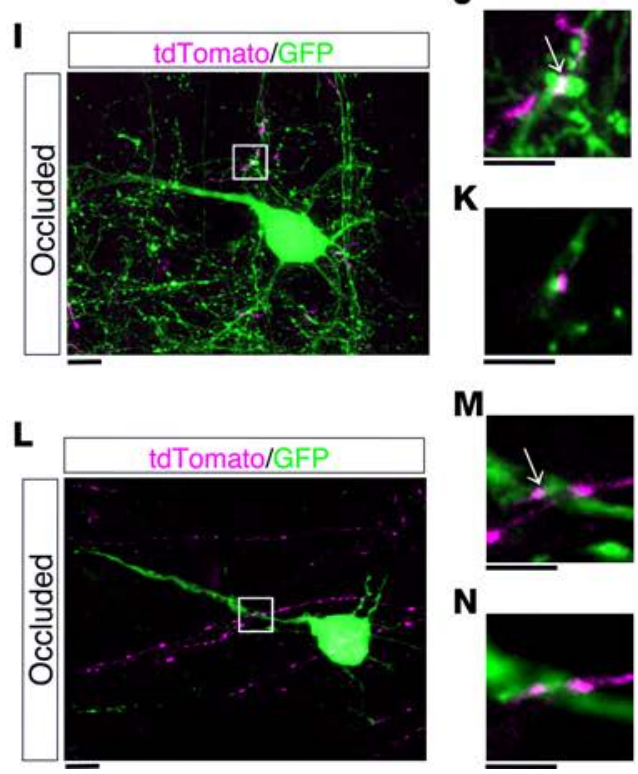

Figure 7. Axonal branching was altered in the brains of the mouse model. (A) Axonal branching of the GFP-positive cells in the contralateral hemisphere in P10 mouse brains that had been transfected with a GFP expression plasmid at E15.0. In the control brains (Control), the GFP-labeled axons branched extensively in the superficial layers (I and II/III). However, in the occluded brains (Occluded), extensive axonal branching was also observed in the deeper layers (layers $\mathrm{V}$ and $\mathrm{VI}$ ). Scale bar: $200 \mu \mathrm{m}$. (B) Fluorescence intensity of GFP in each layer was quantified to analyze the contralateral axonal branching. The GFP intensity was significantly reduced in layers I and II/III and increased in layer $V$ in the occluded brains as compared with the control brains ( $n=6$, respectively). ${ }^{*} P<0.05,{ }^{*} P<0.01,{ }^{* * *} P<0.001$, repeated-measures ANOVA followed by Bonferroni post-hoc test. (C) GFP-positive axons from the contralateral neocortex branched around the abnormally distributed BrdU-positive cells in layer V of P10 occluded mouse brains (arrows). Scale bar: $20 \mu \mathrm{m}$. (D) Schematic representation showing the procedure of in utero electroporation of the right and left neocortices. To visualize the axonal projections from the right neocortex (contralateral hemisphere), a tdTomato expression plasmid was transfected into the right neocortex at E16.5 and a GFP expression plasmid 
was immediately transfected into the left neocortex, followed by a sham operation (Control) or maternal uterine artery occlusion (Occluded). (E) The brains were analyzed at P17.5. Sections were counterstained with DAPI. Some of the GFP-positive cells (green) were distributed in the deeper layers and the white matter in the occluded brains. While the tdTomato-labeled axons (magenta) from the contralateral hemisphere made extensive branching in the superficial layers of the control brains, abundant axonal branching was also observed in the deeper layers of the occluded brains (bottom, indicated by arrows). (F-N) The boxes in $\mathbf{E}$ are shown at high magnification in $\mathbf{F}, \mathbf{I}$, and $\mathbf{L}$, respectively. High-magnification images of the boxes in $\mathbf{F}$, I, and $\mathbf{L}$ are shown in $\mathbf{G}$, J, and $\mathbf{M}$, respectively. In $\mathbf{F}$ and $\mathbf{G}, 25$ confocal planes are merged. In I and J, 22 confocal planes are merged. In $\mathbf{L}$ and $\mathbf{M}$, 21 confocal planes are merged. Single confocal sections of $\mathbf{G}, \mathbf{J}$, and $\mathbf{M}$ are shown in $\mathbf{H}, \mathbf{K}$, and $\mathbf{N}$, respectively. Arrows in $\mathbf{G}, \mathbf{J}$, and $\mathbf{M}$ indicate contacts between the tdTomato-labeled axons and the GFP-labeled dendrites. Scale bar: $10 \mu \mathrm{m}(\mathbf{F}, \mathbf{I}$, and $\mathbf{L}) ; 5 \mu \mathrm{m}(\mathbf{G}, \mathbf{H}, \mathbf{J}, \mathbf{K}, \mathbf{M}$, and $\mathbf{N})$.

When we differentially visualized the normally/ectopically distributed neurons in the neocortex by transfecting GFP plasmid and the axons from the contralateral hemisphere by transfecting tdTomato plasmid (Figure 7D), the tdTomato-labeled axons branched extensively in the superficial layers of the contralateral hemisphere and showed contacts with the normally distributed GFP-positive cells in the control brains (Figure 7, E, top, and F-H). However, in the occluded brains, the tdTomato-labeled axons also made abundant branching in the deeper layers (Figure 7E, bottom, arrows) and showed contacts with the ectopically distributed GFP-positive cells (Figure 7, I-N), further indicating that normal corticocortical axonal branching was impaired in the occluded mouse brains.

The mouse model showed cognitive impairments. Since the pathological evidence of damage to the neuronal progenitor/precursor cells and abnormal ectopic neurons was similar to the findings observed in the extremely preterm infant brains that exhibited WMI, we investigated whether our mouse model would exhibit abnormal behavior at 6 to 7 weeks of age. There were no differences in performance in the openfield test between the occluded group and control group (Supplemental Figure 13A) and no significant differences in locomotor activity between the two groups (Supplemental Figure 13B), suggesting the absence of any significant differences in the motor activities. Furthermore, there were no significant differences in the performance in the light-dark test between the two groups (Supplemental Figure 13C), suggesting the absence of any differences in the anxiety levels, which was also lent support by the absence of any significant difference in the time spent in the inner zone between the two groups in the open-field test (Supplemental Figure 13A).

We then compared the memory performances of the occluded group and control group in the novel object recognition test (Figure 8, A and B). There was no biased exploratory index during the training session (Figure $8 \mathrm{~B}$ ), and both the occluded group and the control group spent equal amounts of time exploring each object (Figure 8B), suggesting no differences in motivation or curiosity in regard to novel objects. However, the level of the exploratory index value in regard to the novel object in the retention session was significantly lower in the occluded group in comparison with the control group (Figure 8B). The total exploration times spent by the two groups in the retention session were not significantly different (Figure $8 \mathrm{~B})$, suggesting that the occluded mice had impaired recognition memory.

Next, to investigate the pathophysiological involvement of the altered neuronal alignment in the occluded brains, we directly manipulated the neural activity of the neurons showing altered alignment during the behavioral tests by using the DREADD (designer receptor exclusively activated by designer drug) system (33, 34) (Figure 8C). We used hM3Dq, a Gq-coupled receptor that responds specifically to clozapine- $N$-oxide (CNO) and induces depolarization of the pyramidal neurons (35). An hM3Dq-expressing vector (pCAGhM3Dq-mCherry) was electroporated with pCAG-EGFP into the right and left mPFCs of mice at E15.0 using the previously established approach of in utero electroporation $(33,36)$ (Figure $8, \mathrm{C}$ and D). We confirmed that the injection of CNO induced elevation of c-Fos expression in the mCherry-positive cells, indicating that it induced excitation of the labeled neurons (Supplemental Figure 14). Disruption of the alignment of the labeled neurons was also confirmed in the mPFCs of the occluded mice (Supplemental Figure 15).

Since various brain regions, including the $\mathrm{MPFC}$ and hippocampus, are thought to be involved in the responses in the novel object recognition test $(37,38)$, we examined spatial working memory using the Y-maze test to address the cognitive functions specific to the neocortex $(39,40)$. Mice expressing hM3Dq in the $\mathrm{mPFC}$ were intraperitoneally injected with $1 \mathrm{mg} / \mathrm{kg} \mathrm{CNO}$ or control saline 1 hour before the task to assess spatial working memory in the presence or absence of CNO (Figure 8E). The occluded mice administered saline showed a significant deficit in spatial working memory (Figure $8 \mathrm{~F}$ ). However, the alternation behavior of the occluded mice in the presence of CNO was comparable to that of the control mice (Figure 8F). These data indicate that activation of the $\mathrm{mPFC}$ in the occluded brains could improve the deficit of spatial working memory, suggesting that the cognitive deficits were caused by decreased circuit activity in the occluded brains. 
A

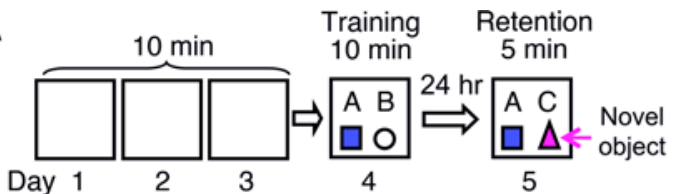

B
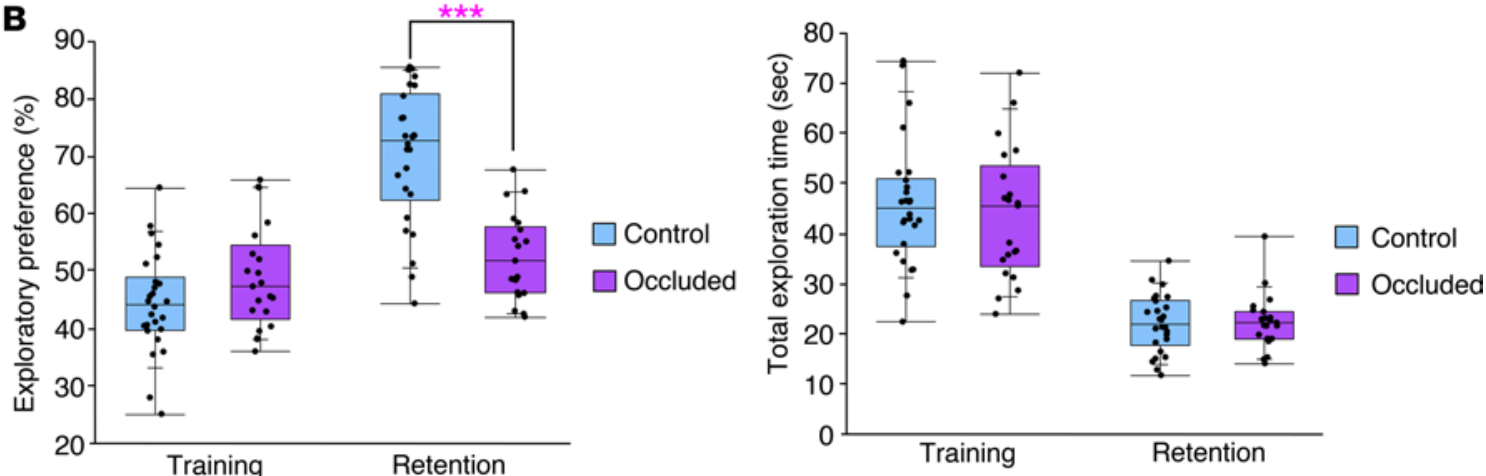

C

EP to MPFC (bilaterally)

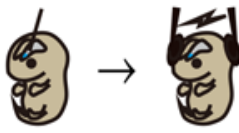

E15
Sham ope (Control group)

$$
\text { or }
$$

$\Rightarrow$ Maternal uterine $\Rightarrow$ arteries occlusion (Occluded group)

E16.5

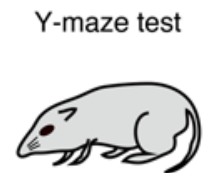

P6-7W
D

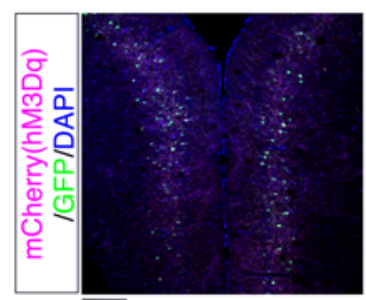

G

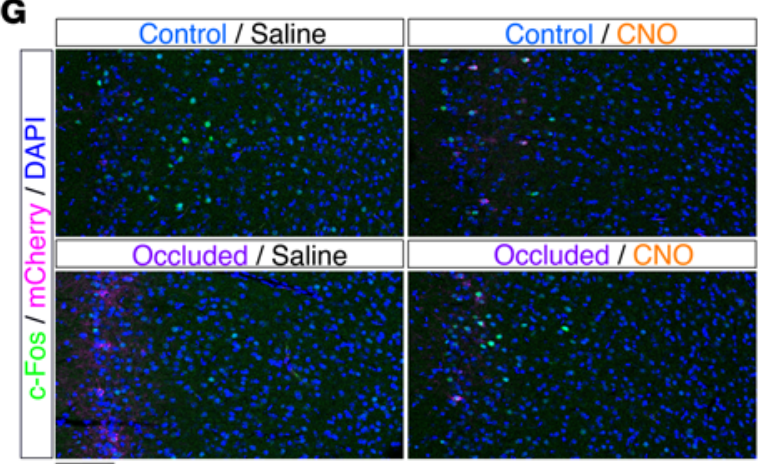

E

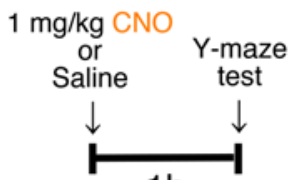

$1 \mathrm{~h}$
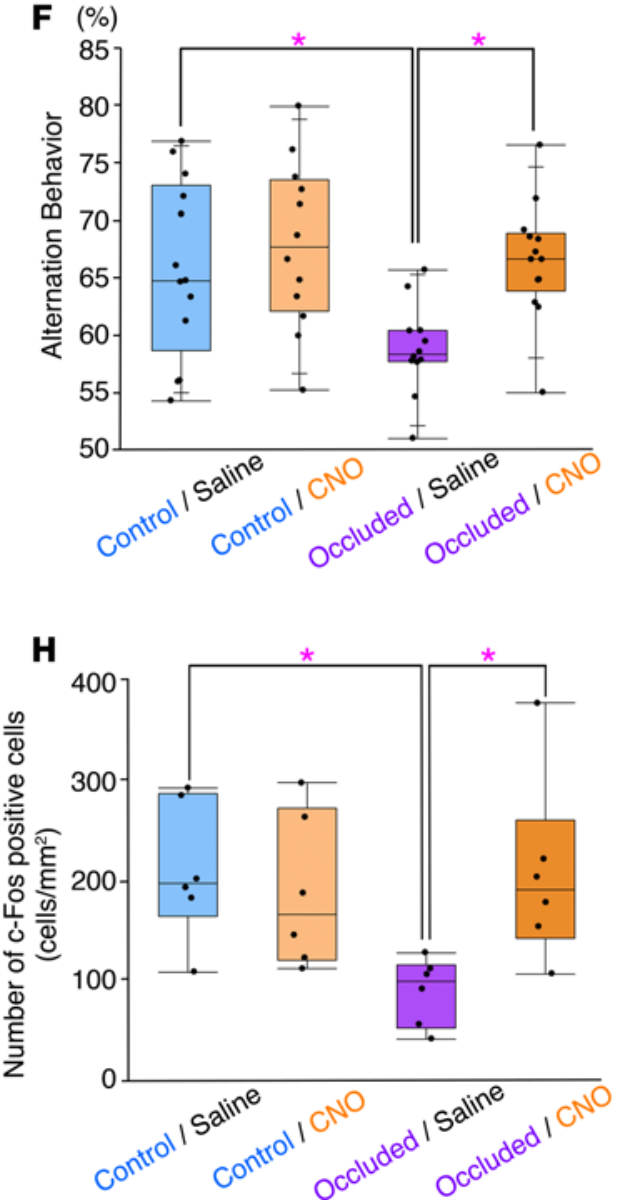

Figure 8. The mouse model had cognitive impairments. (A) Schematic representation of the novel object recognition test. (B) Performance in the novel object recognition test (Control: $n=26$, Occluded: $n=21$ ). Left: exploratory index. Right: total exploration time. ${ }^{* * *} P<0.001$, repeated-measures ANOVA followed by Bonferroni post-hoc test. (C) Schematic representation of bilateral in utero electroporation into the mPFC at E15.0. An hM3Dq-mCherryexpressing vector (pCAG-hM3Dq-mCherry) was coelectroporated with pCAG-EGFP, followed by a sham operation (control) or maternal uterine artery occlusion (Occluded) at E16.5. (D) A representative image of the MPFC transfected with a GFP expression vector and hM3Dq-mCherry expression vector at E15.0 and fixed at P7 weeks. The section was stained with anti-RFP (magenta) antibody. Scale bar: $200 \mu \mathrm{m}$. (E) Scheme showing the time course of the injection of $\mathrm{CNO}$ or control saline and the behavioral test. $(\mathbf{F})$ The graph indicates the spontaneous alternation behavior in the $\mathrm{Y}$-maze test of the control/saline $(n=$ 13), control/CNO ( $n=12)$, occluded/saline $(n=12)$, and occluded/CNO $(n=13)$ mice. ${ }^{*} P<0.05$, Tukey-Kramer test. (C) c-Fos expression in the prelimbic (PrL) region of the $\mathrm{mPFC}$ was analyzed by immunohistochemistry in the saline- and CNO-treated mice 2 hours after the $\mathrm{Y}$-maze test. Images show representa- 
tive examples of c-Fos expression in the PrL. Scale bar: $100 \mu \mathrm{m}$. (H) Quantitative analysis of the number of c-Fos-positive cells in the PrL from the control/ saline, control/CNO, occluded/saline, and occluded/CNO mice ( $n=6$, respectively). ${ }^{*} P<0.05$, Tukey-Kramer test. (B, F, and $\left.\mathbf{H}\right)$ Each point represents an individual mouse. Box-and-whisker plots were used to graphically represent the median (line within box), upper and lower quartiles (bounds of box), and maximum and minimum values (top and bottom bars).

To examine whether the neuronal activity was altered in the $\mathrm{MPFC}$, we investigated the c-Fos expression in the mPFC (Figure $8 \mathrm{G}$ ). The number of c-Fos-positive cells was significantly decreased in the $\mathrm{mPF}$ Cs of the occluded brains but increased in the mPFCs of the occluded brains expressing hM3Dq after CNO treatment (Figure $8 \mathrm{H}$ ); these findings indicate a decrease in the neuronal activity of the occluded brains and an increase in the mPFC activity in the occluded brains expressing hM3Dq after CNO treatment. Consistent with these findings, CNO treatment tended to increase the $\theta$-band $(4-8 \mathrm{~Hz})$ activities of the local field potentials of the mPFCs in the occluded brains, which was significantly diminished in the mPFCs of the occluded brains as compared with the control brains (Supplemental Figure 16).

Maternal mild hypothermia mitigated the neurodevelopmental damage. During the creation of our model, we noticed that the brain injuries were temperature sensitive and that the maintenance of uterine temperature was essential for the induction of brain damage because the uterine temperature could promptly drop during the operations. Clinically, for better survival, the body temperature of extremely preterm infants is maintained at $36.5^{\circ} \mathrm{C}-37.5^{\circ} \mathrm{C}$, which is close to the intraperitoneal condition $(41,42)$. Thus, in general, human extremely preterm infants are thought to be suffered from brain injuries at a temperature of around $36.5^{\circ} \mathrm{C}-37.5^{\circ} \mathrm{C}$. Therapeutic hypothermia is not usually applied to extremely preterm infants, but it has been widely introduced to prevent perinatal encephalopathy in more mature newborns by targeting rectal temperature at $33^{\circ} \mathrm{C}-34^{\circ} \mathrm{C}(43,44)$, which is $3^{\circ} \mathrm{C}-4^{\circ} \mathrm{C}$ lower than the usual target temperature of extremely preterm infants.

In this study, we examined the effect of hypothermia on the brain in our mouse model by changing the temperature of the warmer in which dams were placed during the operation (Supplemental Figure 17). When dams were maintained on a warmer set at $37.5^{\circ} \mathrm{C}$ during the occlusion of their uterine arteries (Supplemental Figure 17A, ii), the surface temperature of uterine horns was about $35^{\circ} \mathrm{C}$ before and after the occlusion (Supplemental Figure 17, B and C). In contrast, when dams were maintained in a mild hypothermia condition on a warmer set at $25.5^{\circ} \mathrm{C}$ during the occlusion (Supplemental Figure 17A, iii), the surface temperature of uterine horns became $31.08^{\circ} \mathrm{C} \pm 0.12^{\circ} \mathrm{C}$, which was $3^{\circ} \mathrm{C}-4^{\circ} \mathrm{C}$ lower than the uterine surface temperature of dams $\left(34.67^{\circ} \mathrm{C} \pm 0.18^{\circ} \mathrm{C}\right.$ ) kept on a warmer set at $37.5^{\circ} \mathrm{C}$ (Supplemental Figure 17 , $\mathrm{B}$ and $\mathrm{C}$ ). Therefore, these procedures mimic the clinical condition of regular and hypothermic treatments in human infants.

When dams were maintained on a warmer set at $25.5^{\circ} \mathrm{C}$, the numbers of $\mathrm{PH} 3$-positive cells in both the $\mathrm{VZ}$ and SVZ of the embryos were significantly higher than in embryos whose mothers were maintained on a warmer set at $37.5^{\circ} \mathrm{C}$ (Figure 9, A and B). Mild maternal hypothermia (on a warmer set at $25.5^{\circ} \mathrm{C}$ ) also significantly mitigated the neuronal migration failure observed in the mothers maintained in the regular condition (Figure 9, C-E). In addition, mild maternal hypothermia significantly rescued the impaired memory performance observed in the animals generated on a warmer set at $37.5^{\circ} \mathrm{C}$ (Figure $9 \mathrm{~F}$ ). Last, we assessed mice using a Y-maze test. The occluded mice generated on a warmer set at $37.5^{\circ} \mathrm{C}$ exhibited significantly less pronounced alternation behavior than the control mice (Figure 9G), and mild maternal hypothermia significantly mitigated the impaired performance (Figure 9G).

\section{Discussion}

In this study, we sought to determine whether brain injury in human extremely preterm infants might influence the migration of neocortical neurons. Although it has been reported that the majority of newly born neurons complete their migration to their cortical targets by 22 GWs (45) and the classical time table indicates that neurogenesis in the human neocortex is completed by the end of the 16th week $(18,19)$, recent studies have yielded compelling evidence to suggest that the VZ persists beyond this time and continues to supply neural cells $(21,22)$. In 23- to 26-GW human fetal brains, we observed neural stem cell marker-positive multilayered cells in the VZ and SVZ (ISVZ and OSVZ) and neuronal marker-positive (HuB, DCX, CUX2, and BRN2) cells having the morphology of migrating neurons in the IZ and SP (Figure 2, C-G, and Supplemental Figure 2), suggesting that neurogenesis and neuronal migration continues even after 23 GWs. Moreover, the outermost region of the human neocortex at 19 

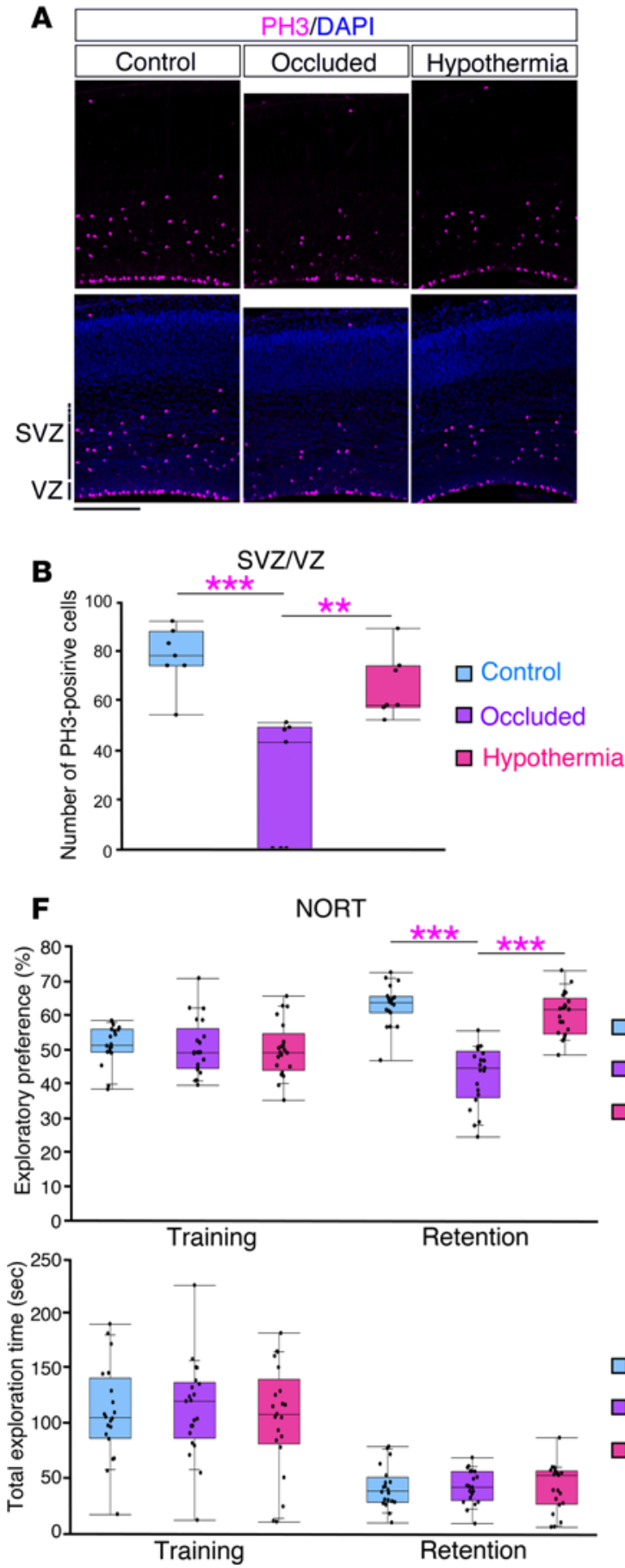
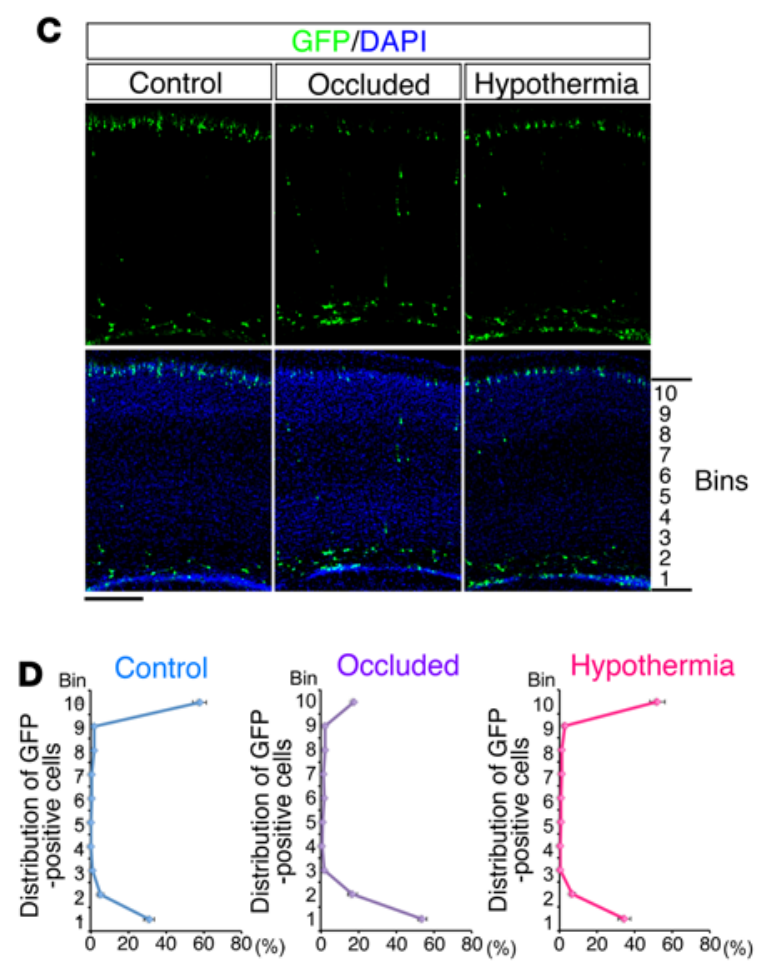

$\mathbf{E}$

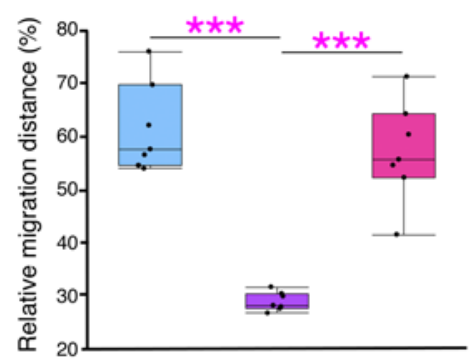

G

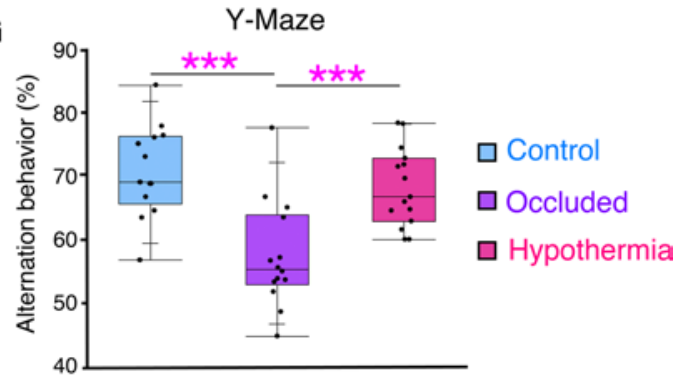

Figure 9. Mild maternal hypothermia mitigated neurodevelopmental damages. (A) A sham operation (Control) or maternal uterine artery occlusion (Occluded and Hypothermia) was performed at E16.5. During the occlusion period, the dam was maintained on a warmer set at a temperature of $37.5^{\circ} \mathrm{C}$ (Occluded) or $25.5^{\circ} \mathrm{C}$ (Hypothermia). Brains were analyzed 12 hours after occlusion. Sections were immunostained with anti-PH3 antibody. Scale bar: 200 $\mu \mathrm{m}$. (B) The numbers of PH3-positive cells in the VZ and SVZ are shown $\left(n=7\right.$, respectively). ${ }^{* *} P<0.01,{ }^{* *} P<0.001$, Tukey-Kramer test. (C) GFP plasmid was transfected at E16.5 and immediately followed by performance of a sham operation (Control) or maternal uterine artery occlusion (Occluded and Hypothermia). During the occlusion period, the dam was maintained on a warmer set at a temperature of $37.5^{\circ} \mathrm{C}$ (Occluded) or $25.5^{\circ} \mathrm{C}$ (Hypothermia) as in A. Brains were analyzed at P3.5. Sections were counterstained with DAPI (blue). Scale bar: $200 \mu \mathrm{m}$. (D) Cell distribution was evaluated by bin analysis. (E) Relative migration distances (\%) from the ventricle are shown $\left(n=7\right.$, each). ${ }^{* *} P<0.001$, Tukey-Kramer test. (F) Performance in the novel object recognition test (NORT). Top: exploratory index. Bottom: total exploration time. Values are mean \pm SEM $\left(n=11\right.$ each). ${ }^{* * *} P<0.001$, repeated-measures ANOVA followed by Bonferroni post-hoc test. (G) The graph indicates the spontaneous alteration behavior in the Y-maze test (Control: $n=13$, Occluded: $n=14$, Hypothermia: $n=15)$. ${ }^{* *} P<0.001$, Tukey-Kramer test. (B, E, F, and $\mathbf{G}$ ) Each point represents an individual mouse. Box-and-whisker plots were used to graphically represent the median (line within box), upper and lower quartiles (bounds of box), and maximum and minimum values (top and bottom bars). 
to $31 \mathrm{GWs}$ was found to be composed of densely packed neurons (Figure 1C and Supplemental Figure $1 \mathrm{~B})$, resembling the PCZ $(23,24)$. The PCZ is thought to be the region in which radially migrating excitatory neurons start to differentiate into mature neurons after completing their migration during the late stage of neocortical development $(23,24)$. Therefore, the existence of this PCZ-like structure at 19 to $31 \mathrm{GWs}$ in the human fetal cortex suggests that radial migration of neocortical neurons is still ongoing near these stages. In addition, CUX1-positive neurons were abundantly distributed throughout the neocortex, including the IZ and SP, at $23 \mathrm{GWs}$, but they were predominantly localized in the cortical plate at 37 GWs (29) (Supplemental Figure 2, C-E), supporting the notion that later-born excitatory neurons continue to migrate even after $23 \mathrm{GWs}$. In the human neocortex, a substantial population of GABAergic neurons is thought to migrate radially from the pallium (46), with the peak migration of the GABAergic neurons occurring around term (40 GWs) (47). Therefore, it is difficult to consider that all the observed CUX1-positive neurons at $23 \mathrm{GWs}$ were GABAergic neurons, since we observed a larger number of CUX1-positive neurons in the SP at $23 \mathrm{GWs}$ than at $37 \mathrm{GWs}$ (Supplemental Figure 2, C-E). Hence, brain injury in extremely preterm infants is assumed to affect the development of the neocortex in which migration of late-born excitatory neurons is still ongoing.

Various factors are considered to cause preterm brain injury, such as asphyxia, ischemia, hypoxia, and inflammation $(5,6,48,49)$. It is supposed that some of these factors, namely, hypoxia (as indicated in Supplemental Table 6) and inflammation (as indicated in Supplemental Table 7 and Supplemental Figure 10) occur simultaneously with or subsequent to occlusion of the maternal arteries in our mouse model. But, our model still does not capture all of these complex etiologies of human extremely preterm infants, and different animal models are required to resolve different questions $(6,48)$. In fact, there are several limitations of our model. First, in this study, we focused on neocortical neuronal migration rather than on other relevant events occurring at a later time, such as the maturation of oligodendrocytes (10) and astrocytes (11), and had to select the stage (E16.5) at which neuronal migration continues in the IZ (the future white matter) in the mouse brains (Figure 4), while having to ignore other events occurring at a later time. In the developing of human brain, neuronal migration of superficial layer neurons might continue disproportionately until the later stages of development, because of the evolutional increase in the number of superficial pyramidal layers $(17,50)$. The second limitation of our model is the phenotypic variation associated with mild-to-severe brain injuries, which resulted in discrepancies between the early and late outcomes due to the death of the most severely affected embryos. This phenotypic variation might be caused, at least in part, by the different susceptibilities of the litters, possibly attributable to their different epigenetic statuses (51). We mainly used mice from a mixed background (ICR mice), and the different genetic backgrounds may also contribute to the variability within a litter. The different positions in the uterus are another possible reason for the variations, since intrauterine position is also known to have profound effects on the behavioral and anatomic traits of a fetus $(52,53)$. On the other hand, phenotypic variations have been observed in other animal models, and these variations are thought to be possibly advantageous, because individual variations occur in humans as well (48).

In the present study, we identified disruption of neural progenitors/precursors and an increase of white matter neurons in an autopsy study of extremely preterm infant brains. These changes in the brains of extremely preterm infants are thought to reflect disruption of neuronal migration, since the brain injuries induced disruption of neuronal alignments (including an increase in the number of white matter neurons) in the mouse model, as demonstrated in the present study. To our knowledge, it was not known until now whether defective neuronal alignment can cause cognitive impairment, but, in this study, we showed that defective neuronal alignment was accompanied by impaired axonal terminal branching (Figure 7 and Supplemental Figure 12), suggesting altered information processing in the occluded mice. In fact, the activation of the mPFC neurons showing abnormal alignment/branching improved the spatial working memory deficit (Figure 8F) and attenuated the decrease in the number of c-Fos -positive cells (Figure $8 \mathrm{H}$ ) observed in the occluded mice, indicating that the cognitive impairment in the occluded mice was caused by decreased mPFC activity. In addition, electrophysiological analysis revealed that the local field potential power in the $\theta$-band was lower in the occluded mice (Supplemental Figure 16), which is consistent with the suggestion that $\theta$ rhythms play important roles in spatial working memory (54). Taken together, we showed that the decreased neuronal activities (especially the $\theta$ rhythms) in the $\mathrm{mPFC}$ with altered neuronal migration were the likely cause of the impaired spatial working memory in the mice, suggesting an association between altered neuronal migration and 
subsequent cognitive impairment, although further studies are required to confirm that altered neuronal migration/alignment can cause decreased neuronal activities in the $\mathrm{MPFC}$. We demonstrated in a previous study that altered neuronal alignment in remote brain regions can cause decreased neuronal activities in the mPFC (33). Thus, the decreased neuronal activities in the mPFC observed in this study might have been caused by alterations of neuronal alignments throughout the cortex (Supplemental Figure 11). Abnormal neuronal alignments have been reported in neuropsychiatric disorders associated with cognitive impairments, such as schizophrenia (55) and autism (56). Interestingly, defective axonal terminal branching that is similar to the impaired corticocortical axonal branching observed in our study has recently been demonstrated in an animal model of schizophrenia (57), lending support to the notion that the impaired corticocortical wiring in brain disorders could cause cognitive impairments.

Another consequence of disrupted neuronal migration is an increase in the number of white matter neurons (Figure 6, C-E). Since brain injuries in extremely preterm infants might also affect the neuronal proliferation that continues after $23 \mathrm{GWs}(21,22)$, the increase in the number of neurons in the white matter might have occurred in a larger scale if there were no reduction in the number of progenitor/precursor cells. The increase in the number of neurons in the white matter may also alter the cortical circuitry, as reported in the cases of intractable epilepsy $(58,59)$, schizophrenia $(60)$, and autism (61). As demonstrated in our present and recent studies (33) (Figure 7), the ectopic neurons in the white matter might trap axons from the contralateral hemisphere and disrupt appropriate axonal extension into the superficial layers of the overlying neocortex. In addition to the ectopic neurons in the white matter, ectopically distributed superficial neurons in the deeper layers are also thought to affect axonal branching, since axons from the contralateral hemisphere seemed to form axonal branches around ectopically distributed cells in the deeper layers (BrdU-positive cells in Figure 7C) and made contact with them (Figure 7, I-N). However, it is difficult to prove at present whether the abnormal pattern of axonal branching was caused by the ectopic location of the neurons. The changes in the axonal branching in the occluded brains in the present study were subtler as compared with those in the brains with the heterotopias observed in a previous study (33). This might reflect the smaller number and more diffuse distribution of the ectopic neurons in occluded brains than in brains with heterotopias (33).

Overall, the observed cognitive impairment in our mouse model is consistent with the high frequency of cognitive impairment observed in the human extremely preterm infants (2-4). In addition to the previously reported mechanisms, including impairments of various cellular components, such as the dendritic arbor and synapse formation of neurons, and myelination failure $(9,13,15)$, defective neuronal alignment is presumed to be another mechanism underlying the later development of cognitive impairment in the extremely preterm infants. Neuronal migration and alignment may also be affected in other brain regions besides the neocortex. Imaging studies of human extremely preterm infants (survivors) have revealed a reduced volume of the deep nuclear gray matter (62), hippocampus (63), and cerebellum (64). We did not find any significant differences in the performance in the light-dark and open-field tests, suggesting the absence of any significant abnormality in anxiety levels, but further behavioral and morphologic studies are required to identify various disturbances in different brain regions. Future studies are also required to reveal the molecular mechanisms underlying the observed neuronal deficits, such as migration delay and abnormal alignment/branching.

Development of reliable mouse models is crucial to test potential therapeutic interventions. In the current study, we found that mild maternal hypothermia efficiently mitigated the decreased proliferation of neuronal progenitors/precursors, deficits of neuronal migration, and the cognitive impairment observed in the occluded mice. In addition to its role in preventing excitotoxicity, apoptosis, inflammation, and free radical production in the brain, hypothermia is thought to affect the blood flow, metabolism, and blood-brain barrier integrity, in addition to the regenerative processes after injury $(49,65)$. Although the effectiveness of hypothermia against the development of perinatal encephalopathy has been clinically demonstrated (44, 66), application of hypothermia to prevent brain injuries in extremely preterm infants remains to be investigated, since concerns regarding adverse effects remain, and intrainsult hypothermia, as used in the present study, would be invasive to the infants and mothers $(67,68)$. Application of therapeutic hypothermia to the extremely preterm infants may not be entirely practical at present, even if only head-specific cooling were considered $(69,70)$. However, it might be possible to modify brain injuries in human extremely preterm infants if the molecular mechanisms underlying the effectiveness of hypothermia were identified, in order to prevent subsequent neurobehavioral complications in extremely preterm infants. 


\section{Methods}

Procedures used for neuropathology, mice, in utero electroporation, maternal uterine artery occlusion, mouse brain slice preparation and staining, BrdU labeling, quantification of cell distribution as well as other quantification, gene microarray analysis, behavioral studies, CNO treatment, and electrophysiological recording as well as the definitions of "severely damaged" and "moderately damaged" brains are described in Supplemental Methods.

Human fetal brain tissue sections from the "Hirata collection." Human fetal brains for tissue sections (Supplemental Table 1) were collected by Yukio Hirata before the 1990s, when he worked at the Tokyo Medical University. Brains were obtained from abortions or autopsies with proper informed consents and were fixed in $10 \%$ formalin in saline. After fixation, most of the brains were processed for celloidin embedding, and microtome sections were cut at 24 to $30 \mu \mathrm{m}$. Nissl staining was carried out with Cresyl violet. At present, the sections are preserved at the University Museum at the University of Tokyo, without any personal information on the subjects. Images were taken with the permission of the museum. Three brains fixed at $23 \mathrm{GWs}$ and two brains fixed at $25 \mathrm{GWs}$ were cut into $40-\mu \mathrm{m}$ sections with a cryostat and used for immunohistochemical staining with primary antibodies against CUX2 (rabbit polyclonal, 1:200, Sigma-Aldrich, SAB2100508), BRN2 (goat polyclonal, 1:50, Santa Cruz, C-20, sc6029), and DCX (goat polyclonal, 1:200, Santa Cruz, C-18, sc8066).

Extremely preterm infant and comparison materials. Comparison (Supplemental Table 2) and extremely preterm infant (Supplemental Table 3) materials were obtained from the autopsy archives of the Department of Pathology of Texas Children's Hospital, Houston, Texas, USA.

Forty-one extremely preterm infant brains with pathologically confirmed WMI were examined (average $25.0 \pm 1.4 \mathrm{GWs}$; ranging from $23 \mathrm{GWs}$ to $27 \mathrm{GWs}$ ). The brains were archival materials from acute cases in which the extremely preterm infants had survived 0-6 days $(n=19$, average birth at $25.2 \pm 1.6$ GWs, duration of survival $1.9 \pm 2.1$ days, corrected age at death $25.2 \pm 1.6 \mathrm{GWs}$ ), subacute cases in which the extremely preterm infants had survived 9-66 days $(n=11$, average birth at $24.6 \pm 1.1$ GWs, duration of survival $28.4 \pm 17.9$ days, corrected age at death $28.3 \pm 3.5 \mathrm{GWs})$, and long-term cases in which the extremely preterm infants survived 4 months to 6 years $(n=11$, average birth at $24.8 \pm 1.2 \mathrm{GWs}$, duration of survival $16.1 \pm 19.9$ months, corrected age at death $12.3 \pm 19.8$ months).

Forty brains total were selected from the archival materials at Texas Children's Hospital after a careful review of the autopsy histories and descriptions of microscopic sections and were used as the comparison brains. Cases in which white matter lesions were recorded were excluded. These brains were of human cases ranging in age from $10 \mathrm{GWs}$ to 21 years and were mainly used as references to understand the developmental stages of human infants (Supplemental Table 2). Of these, we also used 5 brains as comparison brains for the histological studies, as shown in Supplemental Table 2.

Statistics. All histological and behavioral assessments were conducted in a randomized and blinded fashion. Groups were unblinded at the end of each experiment before statistical analyses. Statistical analyses were performed with the software Statcel2 and SPSS Statistics. Data are presented as boxand-whisker plots, which were generated using JMP 12 software. The distribution of the data in each set of experiments was tested for normality. No significant differences in variance between groups were detected using an $F$ test, except for in Figure 3C, Figure 6E, and Supplemental Figure 6F. Student's $t$ test (2 tailed) was used to test for statistically significant differences between the two groups of data in Figure 5G $(P=0.00257)$ as well as in Supplemental Figure 4E ( $P$ values are shown in the legend), Supplemental Figure 9B ( $P=2.11 \mathrm{e}-05)$, Supplemental Figure 13 ( $P$ values are shown in the legend), and Supplemental Figure 17C $(P=2.13 \mathrm{e}-028)$. Welch's $t$ test was used in Figure 3C (MAP2: $P=9.84 \mathrm{e}-09$, NeuN: $P=6.50 \mathrm{e}-011)$, Figure $6 \mathrm{E}$ (BrdU: $P=3.35 \mathrm{e}-05$, NeuN: 3.91e-05), and Supplemental Figure $6 \mathrm{~F}$ $(P=1.52 \mathrm{e}-010)$. Mann-Whitney $U$ test was used in Supplemental Figure 16C $(P=0.0121)$, Supplemental Figure 16D $(P=0.0424)$, and Supplemental Figure 16E $(P=0.230)$. Wilcoxon signed-rank test was used for exploratory analysis in Supplemental Figure 16F (occluded: $P=0.0180$, control: $P=$ 0.144). A Kruskal-Wallis test followed by Dunnett's post-hoc test was used in Figure 5D $(P=3.13 \mathrm{e}-06$, m: $P=3.03 \mathrm{e}-05$, severe: $P=1.75 \mathrm{e}-09)$. ANOVA followed by the Tukey-Kramer test was used to test for the statistical significance of the data in Figure $8 \mathrm{~F}(P=0.00367)$, Figure $8 \mathrm{H}(P=0.0253)$, Figure 9B $(P=1.61 \mathrm{e}-04)$, Figure 9E $(P=2.32 \mathrm{e}-07)$, Figure 9G $(P=4.13 \mathrm{e}-05)$, and Supplemental Figure $4 \mathrm{C}$ $(P=1.94 \mathrm{e}-04)$. Repeated-measures ANOVA followed by Bonferroni post-hoc test was used in Figure 5F $(P=4.30 \mathrm{e}-09$, Bin1: $P=0.00150$, Bin5: $P=0.000550)$, Figure 6A (GFP: $P=6.16 \mathrm{e}-08$, GFP-Bin8: 
$P=0.00691$, GFP-Bin10: $P=0.0211$, BrdU: $P=2.36 \mathrm{e}-08$, BrdU-Bin1: $P=0.000978$, BrdU-Bin2: $P$ $=0.00603$, BrdU-Bin3: $P=0.00713$, BrdU-Bin10: $P=0.0331)$, Figure 7B $(P=0.000730$, layer $\mathrm{I}: P=$ 0.0372, layer II/III: $P=0.00303$, layer V: $P=0.0473$ ), Figure $8 \mathrm{~B}$ (exploratory preference: $P=5.84 \mathrm{e}-$ 08, training: $P=0.0989$, retention: $P=3.43 \mathrm{e}-07$, total exploration time: $P=0.465$, training: $P=0.569$, retention: $P=0.826$ ), Figure $9 \mathrm{~F}$ (exploratory preference: $P=2.68 \mathrm{e}-07$, control vs. occluded: $P=1.16 \mathrm{e}-$ 12, occluded vs. hypothermia: $P=8.10 \mathrm{e}-11$, total exploration time: $P=0.741)$, Supplemental Figure 12B $(P=0.00549$, layer II/III: $P=0.00755$, layer V: $P=0.0109)$, and Supplemental Figure $16 \mathrm{~F}(P=$ 0.145). The relationship between the number of GFAP-positive cells and the number of MAP2-positive cells was measured by calculation of the Pearson product-moment correlation coefficient, as shown in Supplemental Figure 6C $(r=0.00169, n=27, P=0.993)$. A $P$ value less than 0.05 was considered significant in all the statistical tests.

Study approval. Human fetal brains from the "Hirata collection" were analyzed after obtaining the approval of the institutional review board (ethics committee) at Keio University School of Medicine. The extremely preterm infant and comparison materials were obtained from the autopsy archives of the Department of Pathology of Texas Children's Hospital, and the materials were used in this study after obtaining written informed consent from parents or guardians and approval by the institutional review board at the National Center of Neurology and Psychiatry. All animal experiments were performed using protocols approved by the institutional animal care and use committees of Keio University, Nagoya University, and Tokyo Medical and Dental University in accordance with institutional guidelines on animal experimentation at Keio University, Nagoya University, Tokyo Medical and Dental University, and the Japanese Government Law Concerning the Protection and Control of Animals and Japanese Government Notification of Feeding and Safekeeping of Animals.

\section{Author contributions}

$\mathrm{KK}$ and $\mathrm{KD}$ designed research, acquired and analyzed data, wrote the manuscript, and acquired the funding. TN, YI, KY, TE, SB, WS, AK, MA, KI, MS, YM, and KH acquired and analyzed data and reviewed the manuscript. MK, CT, KFT, KT, ST, MN, MI, and YH provided essential tools or materials and contributed to the writing of the manuscript. BA, DDA, and KY provided essential materials, acquired and analyzed data, and contributed to the writing of the manuscript. $\mathrm{KI}$ and $\mathrm{KN}$ designed research, interpreted the data, wrote the manuscript, and acquired the funding.

\section{Acknowledgments}

We thank members of the Nakajima laboratory for valuable discussions. We thank Hideyuki Okano (Keio University School of Medicine) and Jun-ichi Miyazaki (Osaka University, Osaka, Japan) for antibodies and the CAG promoter, respectively. This project was supported by grants from the Strategic Research Program for Brain Sciences ("Understanding of molecular and environmental bases for brain health"); Grantin-Aid for Scientific Research of the Ministry of Education, Culture, Sports, Science, and Technology of Japan; grants from the Japan Society for the Promotion of Science (JP26430075, JP25116522, JP15H01293, JP16K09997, JP24591615, JP15K09723, JP15H02355, JP15H01586, JP16H06482, JP16K14567); Health and Labour Sciences Research Grants; the Takeda Science Foundation; the Naito Foundation; the Keio Gijuku Fukuzawa Memorial Fund for the Advancement of Education and Research; Keio Gijuku Academic Development Funds; and the Program for the Advancement of Keio Next Generation Research Projects.

Address correspondence to: Kazunori Nakajima or Ken-ichiro Kubo, Department of Anatomy, Keio University School of Medicine, 35 Shinanomachi, Shinjuku-ku, Tokyo 160-8582, Japan. Phone: 81.3.5363.3743; E-mail: kazunori@keio.jp (K. Namajima); kkubo@keio.jp (K. Kubo). Or to: Ken Inoue, Department of Mental Retardation and Birth Defect Research, National Institute of Neuroscience, National Center of Neurology and Psychiatry, 4-1-1 Ogawahigashi, Kodaira, Tokyo 187-8502, Japan. Phone: 81.42.346.1713; E-mail: kinoue@ncnp.go.jp.

1. Batton B, Burnett C, Verhulst S, Batton D. Extremely preterm infant mortality rates and cesarean deliveries in the United States. Obstet Gynecol. 2011;118(1):43-48.

2. Marlow N, Wolke D, Bracewell MA, Samara M, EPICure Study Group. Neurologic and developmental disability at six years of age after extremely preterm birth. N Engl J Med. 2005;352(1):9-19. 
3. Johnson S, et al. Neurodevelopmental disability through 11 years of age in children born before 26 weeks of gestation. Pediatrics. 2009;124(2):e249-e257.

4. Hintz SR, et al. Early-childhood neurodevelopmental outcomes are not improving for infants born at $<25$ weeks' gestational age. Pediatrics. 2011;127(1):62-70.

5. Volpe JJ. Brain injury in premature infants: a complex amalgam of destructive and developmental disturbances. Lancet Neurol. 2009;8(1):110-124.

6. Kinney HC, Volpe JJ. Modeling the encephalopathy of prematurity in animals: the important role of translational research. Neurol Res Int. 2012;2012:295389.

7. Deguchi K, Oguchi K, Takashima S. Characteristic neuropathology of leukomalacia in extremely low birth weight infants. Pediatr Neurol. 1997;16(4):296-300.

8. Khwaja O, Volpe JJ. Pathogenesis of cerebral white matter injury of prematurity. Arch Dis Child Fetal Neonatal Ed. 2008;93(2):F153-F161.

9. Buser JR, et al. Arrested preoligodendrocyte maturation contributes to myelination failure in premature infants. Ann Neurol. 2012;71(1):93-109.

10. Back SA, Miller SP. Brain injury in premature neonates: A primary cerebral dysmaturation disorder? Ann Neurol. 2014;75(4):469-486

11. Salmaso N, Jablonska B, Scafidi J, Vaccarino FM, Gallo V. Neurobiology of premature brain injury. Nat Neurosci. 2014;17(3):341-346.

12. Molnár Z, Rutherford M. Brain maturation after preterm birth. Sci Transl Med. 2013;5(168):168ps2.

13. Dean JM, et al. Prenatal cerebral ischemia disrupts MRI-defined cortical microstructure through disturbances in neuronal arborization. Sci Transl Med. 2013;5(168):168ra7.

14. Komitova M, et al. Hypoxia-induced developmental delays of inhibitory interneurons are reversed by environmental enrichment in the postnatal mouse forebrain. J Neurosci. 2013;33(33):13375-13387.

15. McClendon E, et al. Prenatal cerebral ischemia triggers dysmaturation of caudate projection neurons. Ann Neurol. 2014;75(4):508-524

16. Raymond M, Li P, Mangin JM, Huntsman M, Gallo V. Chronic perinatal hypoxia reduces glutamate-aspartate transporter function in astrocytes through the Janus kinase/signal transducer and activator of transcription pathway. J Neurosci. 2011;31(49):17864-17871.

17. Marín-Padilla M. Ontogenesis of the pyramidal cell of the mammalian neocortex and developmental cytoarchitectonics: a unifying theory. J Comp Neurol. 1992;321(2):223-240.

18. Bayer SA, Altman J, Russo RJ, Zhang X. Timetables of neurogenesis in the human brain based on experimentally determined patterns in the rat. Neurotoxicology. 1993;14(1):83-144

19. Golan H, Huleihel M. The effect of prenatal hypoxia on brain development: short- and long-term consequences demonstrated in rodent models. Dev Sci. 2006;9(4):338-349.

20. Vannucci RC, Vannucci SJ. Perinatal hypoxic-ischemic brain damage: evolution of an animal model. Dev Neurosci. $2005 ; 27(2-4): 81-86$

21. Malik S, et al. Neurogenesis continues in the third trimester of pregnancy and is suppressed by premature birth. $J$ Neurosci. 2013;33(2):411-423

22. Zecevic N, Chen Y, Filipovic R. Contributions of cortical subventricular zone to the development of the human cerebral cortex. J Comp Neurol. 2005;491(2):109-122.

23. Sekine K, Honda T, Kawauchi T, Kubo K, Nakajima K. The outermost region of the developing cortical plate is crucial for both the switch of the radial migration mode and the Dab1-dependent "inside-out" lamination in the neocortex. J Neurosci. 2011;31(25):9426-9439.

24. Sekine K, Kubo K, Nakajima K. How does Reelin control neuronal migration and layer formation in the developing mammalian neocortex? Neurosci Res. 2014;86:50-58.

25. Okano HJ, Darnell RB. A hierarchy of Hu RNA binding proteins in developing and adult neurons. J Neurosci. 1997;17(9):3024-3037.

26. Gleeson JG, Lin PT, Flanagan LA, Walsh CA. Doublecortin is a microtubule-associated protein and is expressed widely by migrating neurons. Neuron. 1999;23(2):257-271.

27. Nieto M, et al. Expression of Cux-1 and Cux-2 in the subventricular zone and upper layers II-IV of the cerebral cortex. J Comp Neurol. 2004;479(2):168-180.

28. Oishi K, Aramaki M, Nakajima K. Mutually repressive interaction between Brn1/2 and Rorb contributes to the establishment of neocortical layer 2/3 and layer 4. Proc Natl Acad Sci USA. 2016;113(12):3371-3376.

29. Saito T, et al. Neocortical layer formation of human developing brains and lissencephalies: consideration of layer-specific marker expression. Cereb Cortex. 2011;21(3):588-596.

30. Banker BQ, Larroche JC. Periventricular leukomalacia of infancy. A form of neonatal anoxic encephalopathy. Arch Neurol. 1962;7:386-410.

31. Volpe JJ. Cerebral white matter injury of the premature infant-more common than you think. Pediatrics. 2003;112(1 Pt 1):176-180.

32. Folkerth RD. Neuropathologic substrate of cerebral palsy. J Child Neurol. 2005;20(12):940-949.

33. Ishii $\mathrm{K}$, et al. Neuronal heterotopias affect the activities of distant brain areas and lead to behavioral deficits. $J$ Neurosci. 2015;35(36):12432-12445.

34. Mizoguchi H, et al. Insular neural system controls decision-making in healthy and methamphetamine-treated rats. Proc Natl Acad Sci USA. 2015;112(29):E3930-E3939.

35. Alexander GM, et al. Remote control of neuronal activity in transgenic mice expressing evolved G protein-coupled receptors. Neuron. 2009;63(1):27-39.

36. Niwa M, et al. Knockdown of DISC1 by in utero gene transfer disturbs postnatal dopaminergic maturation in the frontal cortex and leads to adult behavioral deficits. Neuron. 2010;65(4):480-489.

37. Nagai T, et al. Dopamine D1 receptors regulate protein synthesis-dependent long-term recognition memory via extracellular 
signal-regulated kinase 1/2 in the prefrontal cortex. Learn Mem. 2007;14(3):117-125.

38. Cohen SJ, Munchow AH, Rios LM, Zhang G, Asgeirsdóttir HN, Stackman RW. The rodent hippocampus is essential for nonspatial object memory. Curr Biol. 2013;23(17):1685-1690.

39. Sarter M, Bodewitz G, Stephens DN. Attenuation of scopolamine-induced impairment of spontaneous alteration behaviour by antagonist but not inverse agonist and agonist beta-carbolines. Psychopharmacology (Berl). 1988;94(4):491-495.

40. Maurice T, Hiramatsu M, Itoh J, Kameyama T, Hasegawa T, Nabeshima T. Behavioral evidence for a modulating role of sigma ligands in memory processes. I. Attenuation of dizocilpine (MK-801)-induced amnesia. Brain Res. 1994;647(1):44-56.

41. Meyer MP, Bold GT. Admission temperatures following radiant warmer or incubator transport for preterm infants <28 weeks: a randomised study. Arch Dis Child Fetal Neonatal Ed. 2007;92(4):F295-F297.

42. Chitty H, Wyllie J. Importance of maintaining the newly born temperature in the normal range from delivery to admission. Semin Fetal Neonatal Med. 2013;18(6):362-368.

43. Shankaran S. Therapeutic hypothermia for neonatal encephalopathy. Curr Treat Options Neurol. 2012;14(6):608-619.

44. Jacobs SE, Berg M, Hunt R, Tarnow-Mordi WO, Inder TE, Davis PG. Cooling for newborns with hypoxic ischaemic encephalopathy. Cochrane Database Syst Rev. 2013;1: CD003311.

45. Sidman RL, Rakic P. Neuronal migration, with special reference to developing human brain: a review. Brain Res. 1973;62(1):1-35.

46. Letinic K, Zoncu R, Rakic P. Origin of GABAergic neurons in the human neocortex. Nature. 2002;417(6889):645-649.

47. Xu G, et al. Late development of the GABAergic system in the human cerebral cortex and white matter. J Neuropathol Exp Neurol. 2011;70(10):841-858.

48. Jantzie LL, Robinson S. Preclinical models of encephalopathy of prematurity. Dev Neurosci. 2015;37(4-5):277-288.

49. Wassink G, Gunn ER, Drury PP, Bennet L, Gunn AJ. The mechanisms and treatment of asphyxial encephalopathy. Front Neurosci. 2014;8:40.

50. Nowakowski TJ, Pollen AA, Sandoval-Espinosa C, Kriegstein AR. Transformation of the radial glia scaffold demarcates two stages of human cerebral cortex development. Neuron. 2016;91(6):1219-1227.

51. Yildirim $\mathrm{F}$, et al. Histone acetylation and CREB binding protein are required for neuronal resistance against ischemic injury. PLoS One. 2014;9(4):e95465.

52. Ryan BC, Vandenbergh JG. Intrauterine position effects. Neurosci Biobehav Rev. 2002;26(6):665-678.

53. Nagao T, et al. Intrauterine position and postnatal growth in Sprague-Dawley rats and ICR mice. Reprod Toxicol. 2004;18(1):109-120.

54. Colgin LL. Mechanisms and functions of theta rhythms. Annu Rev Neurosci. 2013;36:295-312.

55. Casanova MF, et al. Neuronal distribution in the neocortex of schizophrenic patients. Psychiatry Res. 2008;158(3):267-277.

56. Bailey A, et al. A clinicopathological study of autism. Brain. 1998;121 (Pt 5):889-905.

57. Mukai J, et al. Molecular substrates of altered axonal growth and brain connectivity in a mouse model of schizophrenia. Neuron. 2015;86(3):680-695.

58. Sisodiya SM. Malformations of cortical development: burdens and insights from important causes of human epilepsy. Lancet Neurol. 2004;3(1):29-38.

59. Manent JB, Wang Y, Chang Y, Paramasivam M, LoTurco JJ. Dcx reexpression reduces subcortical band heterotopia and seizure threshold in an animal model of neuronal migration disorder. Nat Med. 2009;15(1):84-90.

60. Connor CM, Crawford BC, Akbarian S. White matter neuron alterations in schizophrenia and related disorders. Int J Dev Neurosci. 2011;29(3):325-334.

61. Wegiel J, et al. The neuropathology of autism: defects of neurogenesis and neuronal migration, and dysplastic changes. Acta Neuropathol. 2010;119(6):755-770.

62. Thompson DK, et al. Perinatal risk factors altering regional brain structure in the preterm infant. Brain. 2007;130(Pt 3):667-677.

63. Nosarti C, Froudist-Walsh S. Alterations in development of hippocampal and cortical memory mechanisms following very preterm birth. Dev Med Child Neurol. 2016;58 Suppl 4:35-45.

64. Weindling M. Insights into early brain development from modern brain imaging and outcome studies. Acta Paediatr. 2010;99(7):961-966.

65. Yenari MA, Han HS. Neuroprotective mechanisms of hypothermia in brain ischaemia. Nat Rev Neurosci. 2012;13(4):267-278.

66. Azzopardi DV, et al. Moderate hypothermia to treat perinatal asphyxial encephalopathy. N Engl J Med. 2009;361(14):1349-1358.

67. Gancia P, Pomero G. Brain cooling and eligible newborns: should we extend the indications? J Matern Fetal Neonatal Med. 2011;24 Suppl 1:53-55.

68. de Almeida MF, et al. Hypothermia and early neonatal mortality in preterm infants. J Pediatr. 2014;164(2):271-5.e1.

69. Hoque N, Liu X, Chakkarapani E, Thoresen M. Minimal systemic hypothermia combined with selective head cooling evaluated in a pig model of hypoxia-ischemia. Pediatr Res. 2015;77(5):674-680.

70. Gunn AJ, et al. Therapeutic hypothermia translates from ancient history in to practice. Pediatr Res. 2017;81(1-2):202-209. 